\title{
Article \\ Bayesian Optimization and Hierarchical Forecasting of Non-Weather-Related Electric Power Outages
}

\author{
Olukunle O. Owolabi ${ }^{1, *(1)}$ and Deborah A. Sunter $1,2,3,4,5, *$ \\ 1 Department of Mechanical Engineering, Tufts University, Medford, MA 02155, USA \\ 2 Department of Civil and Environmental Engineering, Tufts University, Medford, MA 02155, USA \\ 3 Department of Computer Science, Tufts University, Medford, MA 02155, USA \\ 4 Center for International Environment and Resource Policy, Tufts University, Medford, MA 02155, USA \\ 5 Tufts Institute of the Environment, Tufts University, Medford, MA 02155, USA \\ * Correspondence: olukunle.oladipo@gmail.com (O.O.O.); deborah.sunter@tufts.edu (D.A.S.)
}

Citation: Owolabi, O.O.; Sunter, D.A. Bayesian Optimization and Hierarchical Forecasting of Non-Weather-Related Electric Power Outages. Energies 2022, 15, 1958. https://doi.org/10.3390/en15061958 Academic Editor: Javier Contreras

Received: 20 January 2022

Accepted: 2 March 2022

Published: 8 March 2022

Publisher's Note: MDPI stays neutral with regard to jurisdictional claims in published maps and institutional affiliations.

Copyright: () 2022 by the authors. Licensee MDPI, Basel, Switzerland. This article is an open access article distributed under the terms and conditions of the Creative Commons Attribution (CC BY) license (https:// creativecommons.org/licenses/by/ $4.0 /)$.

\begin{abstract}
Power outage prediction is important for planning electric power system response, restoration, and maintenance efforts. It is important for utility managers to understand the impact of outages on the local distribution infrastructure in order to develop appropriate maintenance and resilience measures. Power outage prediction models in literature are often limited in scope, typically tailored to model extreme weather related outage events. While these models are sufficient in predicting widespread outages from adverse weather events, they may fail to capture more frequent, non-weather related outages (NWO). In this study, we explore time series models of NWO by incorporating state-of-the-art techniques that leverage the Prophet model in Bayesian optimization and hierarchical forecasting. After defining a robust metric for NWO (non-weather outage count index, NWOCI), time series forecasting models that leverage advanced preprocessing and forecasting techniques in Kats and Prophet, respectively, were built and tested using six years of daily stateand county-level outage data in Massachusetts (MA). We develop a Prophet model with Bayesian True Parzen Estimator optimization (Prophet-TPE) using state-level outage data and a hierarchical Prophet-Bottom-Up model using county-level data. We find that these forecasting models outperform other Bayesian and hierarchical model combinations of Prophet and Seasonal Autoregressive Integrated Moving Average (SARIMA) models in predicting NWOCI at both county and state levels. Our time series trend decomposition reveals a concerning trend in the growth of NWO in MA. We conclude with a discussion of these observations and possible recommendations for mitigating NWO.
\end{abstract}

Keywords: electrical power outage; non-weather outages; Prophet model; hierarchical forecasting; Bayesian optimization

\section{Introduction}

Power outages demonstrate a failure in the proper functioning of an electrical distribution system [1-4]. These outage events can result in substantial financial losses [5-7], such as food spoilage [8,9] or a serious health emergency in a health facility [10], especially when back-up sources of generation fail [11]. Developing a robust outage risk mitigation strategy [12,13] is important for utility managers to formulate protective and preventive measures that reduce the occurrence of electric outages. Outage forecasting models are a useful tool for uncovering historical and future trends of outage events and can, therefore, guide outage preventive and mitigation strategies [14].

The cause of an outage influences its magnitude or severity. Outages as a result of extreme weather events (Extreme Weather Outages, EWO), such as a storm or high winds, may result in a loss of power for numerous customers at a time [15-17]. Outages unassociated with adverse weather events (Non-extreme weather outages or non-weather related outages, NWO) often affect a smaller proportion of electricity customers at a time, such as those emanating from the ecology domain as a result of an animal disturbance [18-20]. 
While in most cases (and as shown in Table 1), the average and median values of the number of customers affected and the duration of outages from EWO often exceed those of NWO, these NWOs have a significant cumulative effect on the electrical distribution grid. As can be seen in the summary statistics in Table 1, the total number of outages and total number of customers affected as a result of NWO in MA exceed those of EWO within the studied 6-year period from 2013-2018. However, it is important to note that the statistics presented in Table 1 are only valid for the study location and period. Large EWO are less frequent and therefore short-term comparison (as in Table 1) gives only partial information about the long term effect of EWO relative to NWO.

Table 1. Comparison of Extreme Weather outages (EWO) and Non-Extreme Weather outages (NWO) in MA based on the outage data from 2013-2018. Source: statistics are based on data obtained from MA department of public utilities [21].

\begin{tabular}{ccc}
\hline & EWO & NWO \\
\hline Average Number of Customers Affected & 144 & 127 \\
Median Number of Customers Affected & 14 & 13 \\
Average Outage Duration (Hours) & 13.60 & 6.85 \\
Median Outage Duration (Hours) & 3.57 & 1.78 \\
Total Number of Outages & 61,173 & 75,671 \\
Total Duration of Outages (Days) & 834,513 & 518,644 \\
Total Number of Customers Affected & $8,810,039$ & $9,680,001$ \\
\hline
\end{tabular}

The majority of outage forecasting models in literature have focused on EWO [14,16,22-37] while neglecting the impact of NWO whose long-term cumulative effects are substantial. There have been a few NWO studies that have focused on subsets of these outages, such as animal and vegetation related outages. For example, the prediction and analysis of animal related outages have been explored using a combination of statistical and advance machine learning techniques $[18,20,38]$. Also, similar predictive techniques for vegetation related outages (as a result of tree growth) have been explored [39].

This study explores NWO and aims to understand trends in these outages that may be useful in improving electrical distribution grid reliability. The specific contribution of this study include: (1) development of a quantitative metric for NWO that can aid the understanding and quantification of these outages, (2) development of a time series forecasting framework that exposes critical trends and seasonal patterns in NWO, and (3) improvement of the state-of-the-art prediction performance using proposed Bayesian and hierarchical methods. Based on the study methodology (Figure 1), we develop a robust time series forecasting framework for NWO at the state and county level. Our case study is based on the state of Massachusetts (MA) with outage data from 2013-2018 [21]. After deriving a quantitative count-based metric (Non-weather Outage Count Index, NWOCI) for NWO and leveraging advanced data preprocessing tools in Kats [40], a Bayesian optimization method with a Prophet model [41] is used for the state level forecast and a hierarchical Prophet bottom-up approach is used to produce the county level forecasts. Bayesian optimization helps to improve model performance and reduce computation time [42], while hierarchical models are the optimal choice for multi-time series forecasting, especially for grouped or subdivided data [43] (in this case, county level forecasting for multiple counties in the study region-MA). A comparative analysis evaluates the performance of the proposed models relative to other SARIMA and Prophet Bayesian and hierarchical models. The forecast trend and seasonality are also analysed to better understand observed historical and future NWO trends. The complete end-to-end methodology used in this study is describe in Figure 1. 


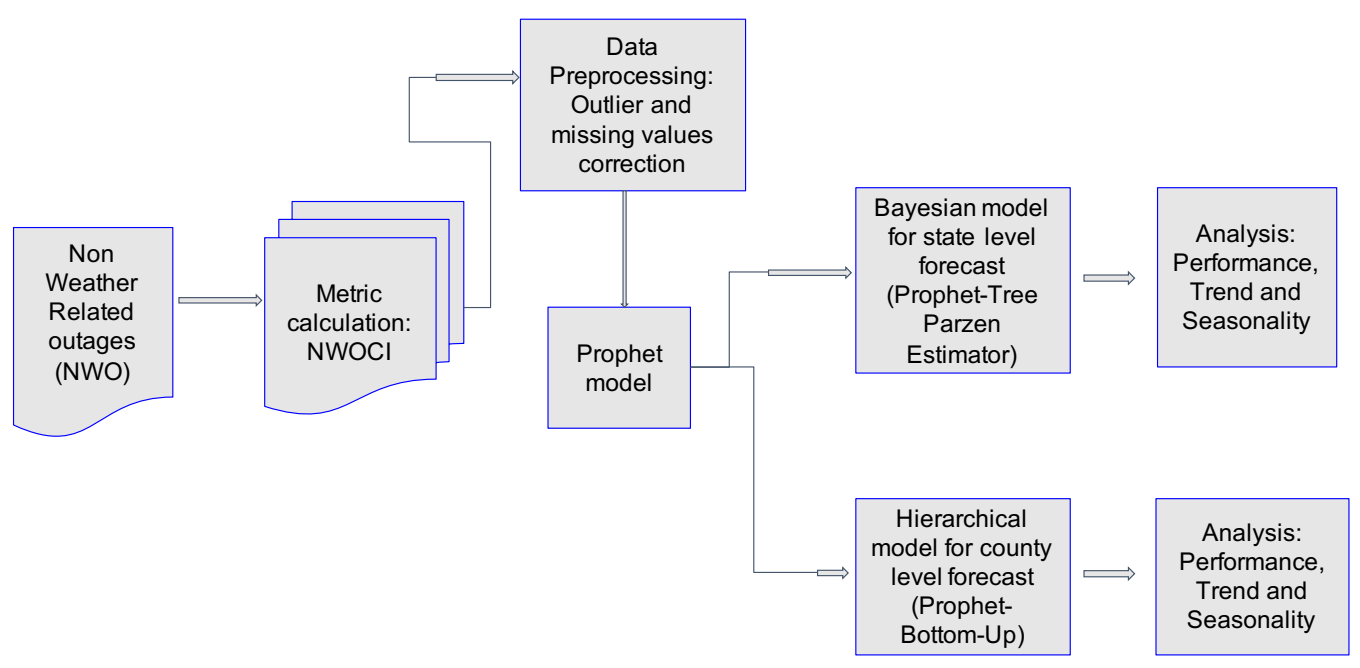

Figure 1. Flow chart of methodology used for this study.

\section{Methods}

\subsection{Data Collection}

\subsubsection{Outage Data}

The state of MA was selected for this study based on the availability of outage reports across the state between 2013-2018. These reports are a component of the Emergency Response plan filed by each of the three major utilities in MA-National Grid, Eversource Energy, and UNITIL corporation-and contain information on the date, time, location, and cause of the outage [21].

Data preprocessing was done to ensure data integrity and develop consistent daily time series. First, data verification procedures were used to address problems such as typographical errors or mismatch in the location reported for the outage. Details of these procedures can be found in the Appendix A.1. Next, steps were taken to obtain a consistent, ready-to-use, daily time series. First, days with no outages in the aggregated time series are replaced with zeros. Then outlier values were treated using the outlier detector algorithm in the Kats toolkit [40] using the standard $1.5 \times(\mathrm{IQR})$ outlier designation and replaced with the interpolated value.

\subsubsection{Weather Data}

Wind speed data (including gust speed) between 2013-2018 (corresponding to the range of the outage data), was collected from all 23 operating weather stations in MA (Figure 2) at hourly level from the National Oceanic and Atmospheric Administration (NOAA) database via the climate data online local climatological data repository [44]. The wind gusts data are only reported when there is a short-term wind speed (typically $20 \mathrm{~s}$ or less) that both exceeds 16 knots (about $18 \mathrm{mph}$ ) and exceeds the average wind speed by at least 9-10 knots (10-11 mph) [45]. Whenever there is a gust speed, we replace the average wind speed value at that hour with the gust speed to account for the influence of these short term extreme winds. The hourly wind speed were matched with the raw outage data corresponding to the starting time (hour) and location (shortest euclidean distance) of the outage event and then used to delineate extreme weather outage instances. 


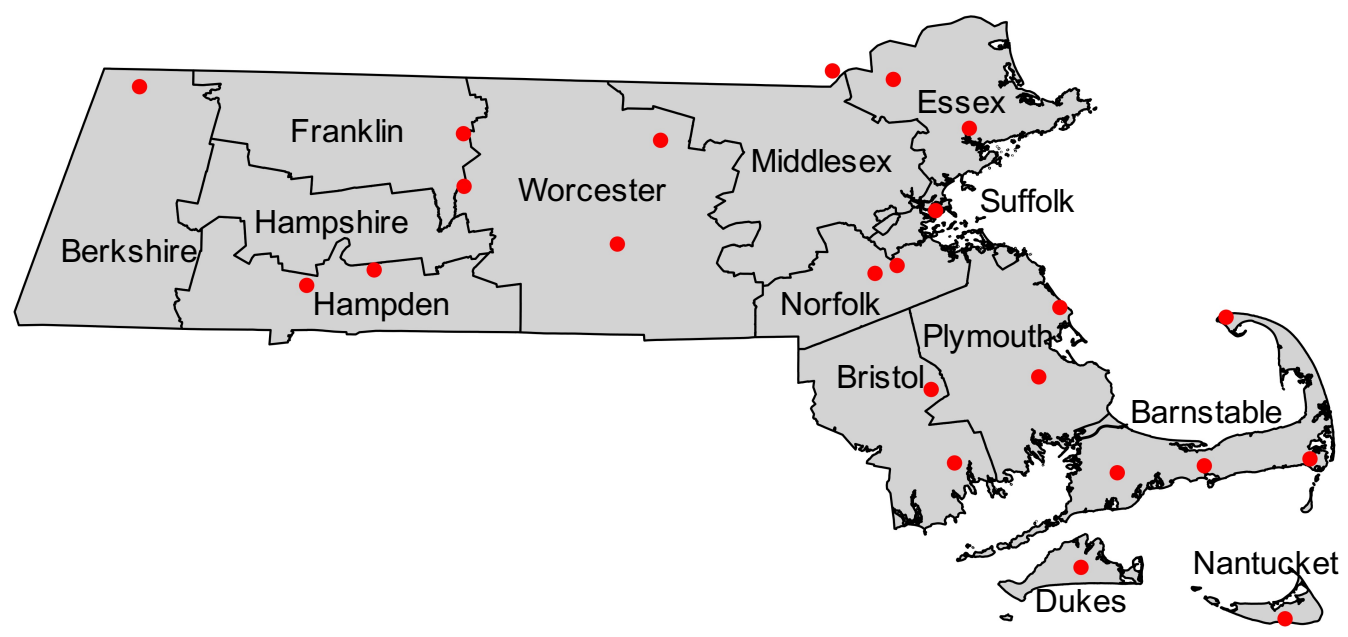

Figure 2. Weather stations in MA where wind speed data was collected. Source: map was developed based on weather station data obtained from NOAA climate data repository [44].

\subsection{Non-Weather Outage Count Index (NWOCI)}

We classify an outage as an NWO if the outage did not occur during instances of high wind threat and other adverse weather conditions including precipitation, lightning, thunderstorm, and snow. First, we begin with classifying Extreme Weather Outages (EWO) based on two indicators:

1. Categorical classification of the outage event-adverse weather conditions (which captures all other extreme weather events including Wind, Precipitation, Snow, Lightning, and Thunderstorm)

2. Wind threat threshold: If the wind speed exceeds $40 \mathrm{mph}-\mathrm{a}$ threshold above which it poses a high or extreme threat, according the National Oceanic and Atmospheric Administration (NOAA) as seen in Table 2 [46], the outage is categorized as an EWO. We use this wind threat to further validate our classification of EWO while capturing potential mis-classified outages.

Table 2. Wind threat threshold as defined by NOAA. Source: Adapted from NOAA [46].

\begin{tabular}{ccc}
\hline Threat Level & Description & Wind Speed (mph) \\
\hline Extreme & An Extreme Threat to Life and Property from High Wind. & $>58$ \\
\hline High & $\begin{array}{c}\text { High wind: } \\
\text { A High Threat to Life and Property from High Wind. }\end{array}$ & $40-57$ \\
\hline Moderate & $\begin{array}{c}\text { Very windy: } \\
\text { Low }\end{array}$ & A Moderate Threat to Life and Property from High Wind. \\
Breezy to windy: & $26-39$ \\
\hline Very low & No Discernable Threat to Life and Property from High Wind. & $21-25$ \\
\hline
\end{tabular}

We then define the daily Non-extreme Weather Outage Count Index (NWOCI) as the total number of NWOs each day in the state and each county, respectively. This is calculated by subtracting the number of outages classified as EWO from the total number of reported outages. It is important to note that each NWO is counted equally, regardless of outage duration and the number of customers affected. As seen in Table 1, the duration and number of affected customers is typically modest for NWO relative to EWO. The focus of this study is on the frequency of NWO and not the magnitude of these events. 


\subsection{Prophet Model}

Prophet [41], an open-source software released by Facebook's Core Data Science team, was used for time series forecasting. Prophet uses a decomposable model architecture including the following model elements: trend $g$, seasonality $s$, and holidays $h$.

$$
y(t)=g(t)+s(t)+h(t)+\varepsilon_{t}
$$

In this study, we use the linear trend model specification in Prophet given as:

$$
g(t)=k t+m
$$

where $k$ is the growth rate and $m$ is the offset parameter; details on the Prophet model can be found in [47].

\subsection{Bayesian Optimization Using True Parzen Estimator (TPE)}

In this study, we incorporated Bayesian optimization into the Prophet time series model, using the True Parzen Estimator (TPE) [48]. TPE is a type of Sequential Model Based Optimization (SMBO) [49] technique that uses the Bayesian approach in an effort to reduce computational time. The implementation of TPE Bayesian optimization in this study was carried out using the hyperopt software package [48] in the Python programming language. We call this integrated model, that uses the Prophet-based objective function with TPE, Prophet-TPE. The steps in developing Prophet-TPE (as illustrated in Figure 3) include:

1. Search domain: First, the domain over which the hyperparameter search will be conducted is defined. For the first iteration, a random combination of hyperparameters within the ranges set forth in Table A1, is used. In each subsequent iteration, the combination of hyperparameters is adjusted using the probability distribution based on the performance of other combinations used in previous iterations.

2. Objective function: The objective function takes in a combination of hyperparameters and output the 5-fold expanding window cross-validated root mean squared error (RMSE) to be minimized over the Prophet model one-year ahead forecast. Unlike a rolling window that moves the upper and lower bound with each time step, the expanding window fixes the lower bound; thus, the amount of data considered incrementally increases (expands) with each time step. The cross validation was performed using the built-in cross validation diagnostic in Prophet [41]. A model is then built to evaluate the objective function. This model is called the surrogate model.

3. History: In Prophet-TPE each iteration forms the history. This set of historical information on the performance of a set of hyperparameters on the actual objective (minimizing the Prophet model's error) is used to construct the probability distributions.

4. Probability distribution: This is a mapping of the probability of error, $y$, for a combination of hyperparameters, $x$.

5. Evaluation criteria: This is the method for obtaining the next best set of hyperparameters. The evaluation criteria is called the Expected Improvement (EI) which is given as [48]:

$$
\begin{gathered}
E I_{y *}(x)=\int_{-\infty}^{y}\left(y^{*}-y\right) P(y \mid x) d y \\
P(y \mid x)=\frac{P(x \mid y)}{P}(y) P(x) \\
P(x \mid y)= \begin{cases}l(x), & \text { if } y<y * \\
g(x), & \text { if } y \geq y *\end{cases}
\end{gathered}
$$

where $g(x)$ and $l(x)$ are probability distributions and $y^{*}$ is the current best set of model hyperparameters in the history (lowest RMSE). The aim is to choose the next hyperparameter combinations that maximizes EI. 
6. Update history: The set of distributions developed from historical information forms the basis of a series of iterative improvement for the surrogate model until the maximum number of iterations $(n=10)$ is reached.

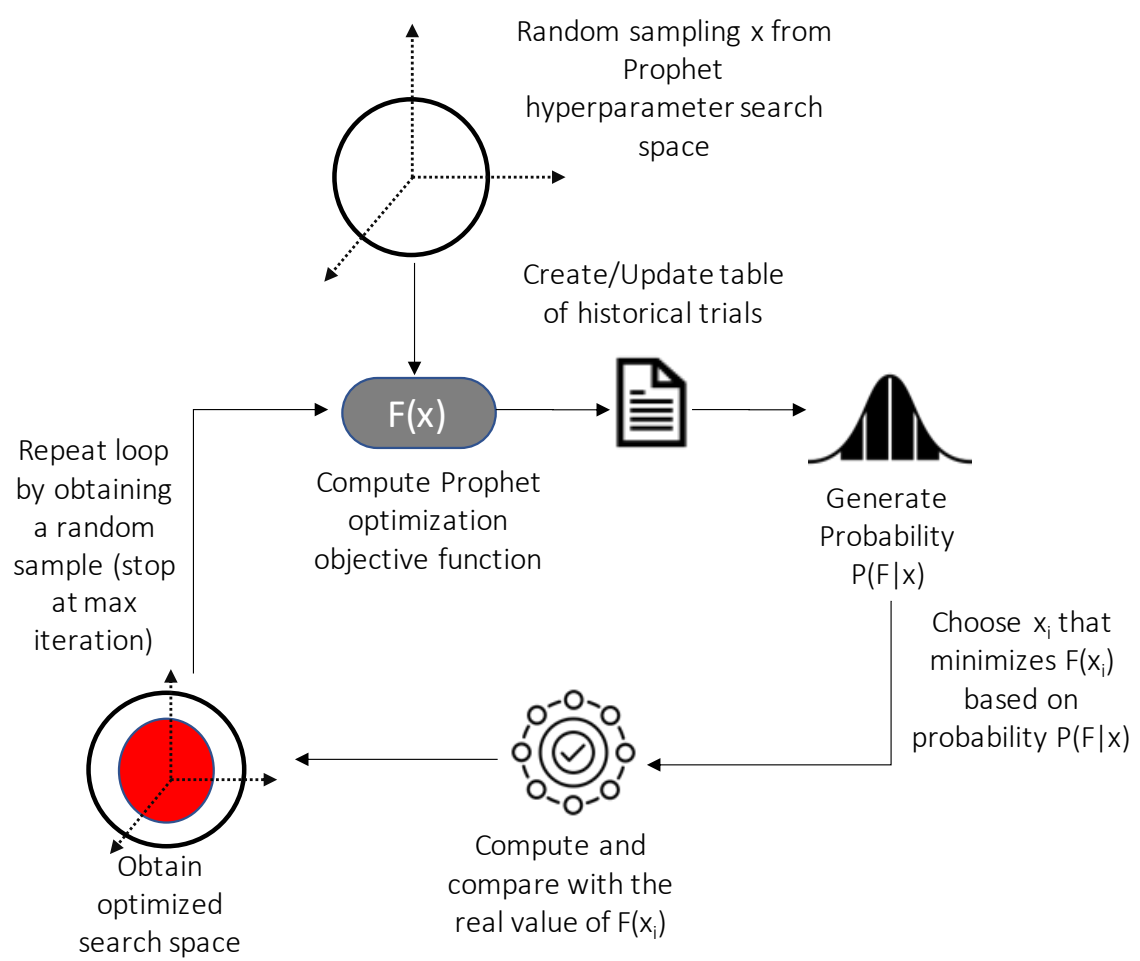

Figure 3. Optimization methodology of the Bayesian True Parzen Estimator with the Prophet-based objective function (Prophet-TPE).

\subsection{Hierarchical Forecasting Model with Bottom-Up Approach}

Hierarchical forecasting is a way to obtain disaggregated, multi-time series forecast at finer scales that must add up to the whole [50]. This approach may improve forecast performance while reducing dis-aggregation error. In the bottom up approach, forecasts are obtained for lower levels in the hierarchy with upward aggregation to reconcile the forecast with the upper levels of the hierarchy. We represent the outage data as a hierarchical tree, with a focus on the county-level forecasts and reconciliation at the state level, as seen in Figure 4. We then propose a Prophet model with bottom up approach (Prophet-BU) for forecasting county-level NWOCI. The Prophet-BU forecasting approach was implemented in this study using the scikit-hts software package [51] in the Python programming language.

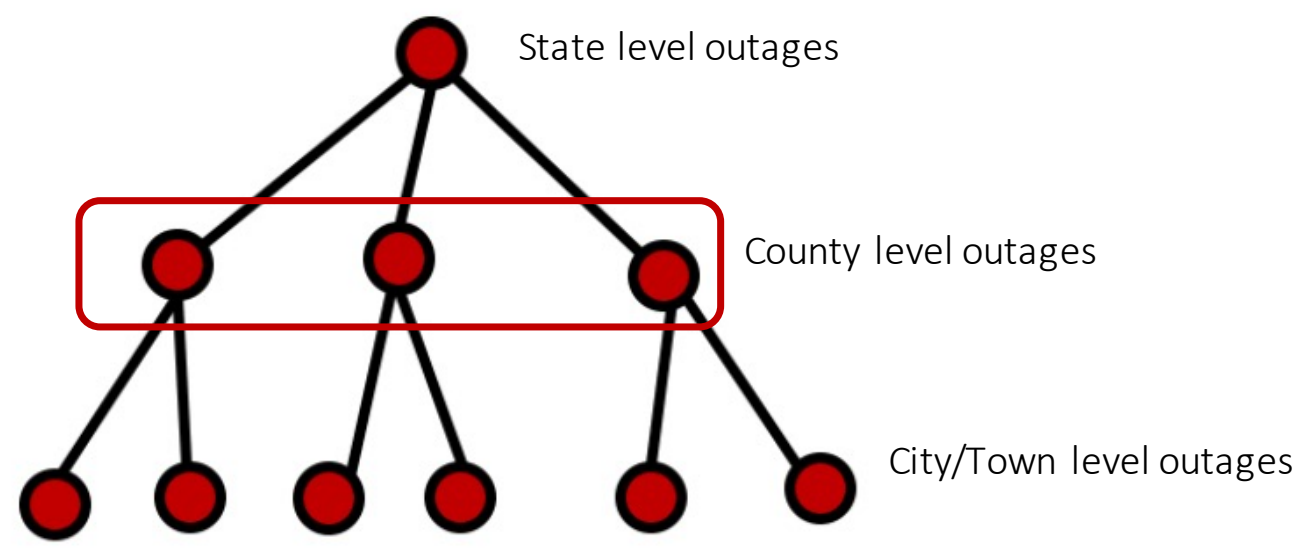

Figure 4. Hierarchical representation of outages. 


\subsection{Performance Evaluation}

The performance of the proposed models is evaluated using the RMSE to assess the difference between the daily forecasted prediction of NWOCI and the actual NWOCI at the state level for Prophet-TPE and the county level for Prophet-BU. Since we have time series data, the data are divided sequentially into train and test with 2013-2017 NWOCI used for training and 2018 data used for testing for both the Prophet-TPE (state level forecast) and the Prophet-BU (county level forecast). The default interval width of 0.8 , representing the $80 \%$ confidence interval, was used to understand the forecast uncertainty of the proposed models.

The performance of the proposed models, Prophet-TPE and Prophet-BU, is compared with other Bayesian and hierarchical model combinations. For the state-level forecast, the proposed Prophet-TPE method is compared with other Seasonal Autoregressive Integrated Moving Average (SARIMA) and Prophet Bayesian models. These include: SARIMATPE, SARIMA-Anneal (SARIMA model with simulated annealing), and Prophet-Anneal (Prophet with simulated annealing). For the county-level forecast, the proposed ProphetBU model is compared with other Prophet and SARIMA heirachical forecasting models. These include the Prophet and SARIMA model in combination with each of the following: AHP (Average Historical Proportion-top down), OLS (Ordinary Least Square revision), WLSS (Structurally Weighted Least Squares revision), FP (Forecasted Proportions-top down), and PHA (Proportion of Historical Averages-top down). A complete list of the models used in the performance evaluation is shown in Figure 5.

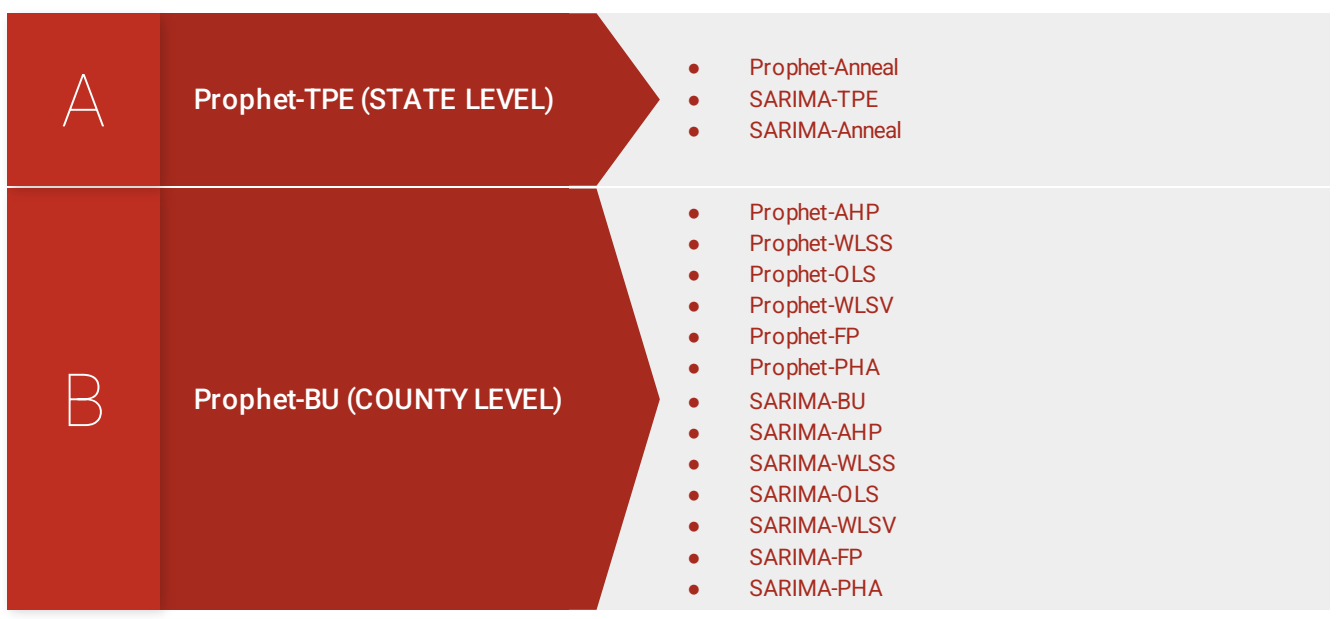

Figure 5. Performance Evaluation of the proposed Prophet-TPE and Prophet-BU models with model combinations of Prophet and SARIMA Bayesian and hierarchical forecasting models.

\section{Results and Finding}

The result for the state level forecast (Table 3) shows the performance of the ProphetTPE in comparison with other models. The optimal hyperparameter values found for the Prophet-TPE and other models are given in Table A2. We observed that the proposed Prophet-TPE gives the best prediction for NWOCI at the state level. The Prophet-TPE forecast results can be seen in Figure 6. A decomposition of the forecast Prophet-TPE trend (Figure 7) reveals a positive linear growth trend in average NWOCI representing a 100\% increase from 2013-2019 (from 17 in 2013 to 34 in 2019). The seasonal decomposition plot of the Prophet-TPE model forecast shows the temporal patterns of NWOCI with a peak in the summer months (July peak). 


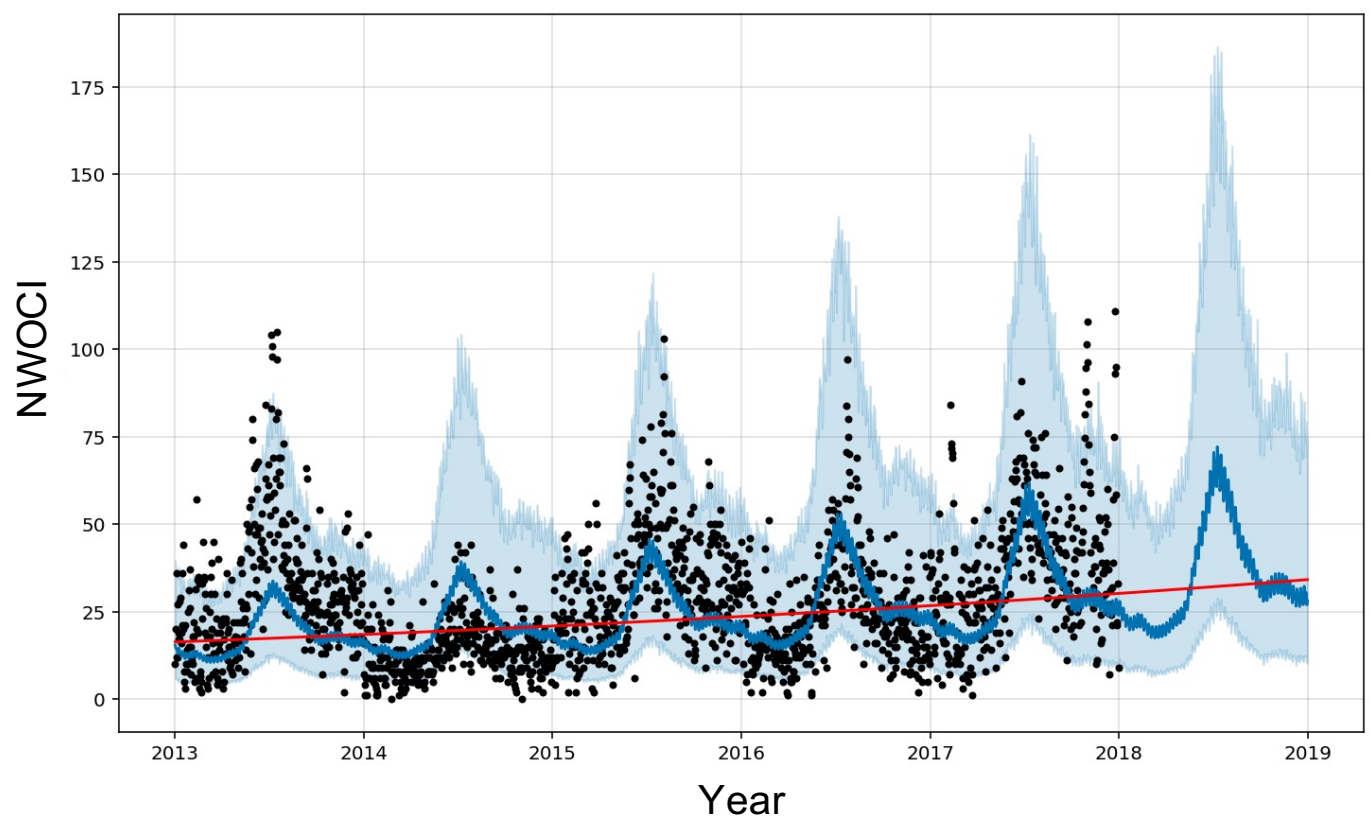

Figure 6. One year forecast result (2018 test data) from Prophet-TPE Model. N.B: The solid blue line shows the average forecast while the solid red line shows the observed trend of NWOCI and the light blue region represents the $80 \%$ confidence interval for the forecast uncertainty. Also, the scatter points represent the historical values (daily state level NWOCI) in the training data (2013-2017).
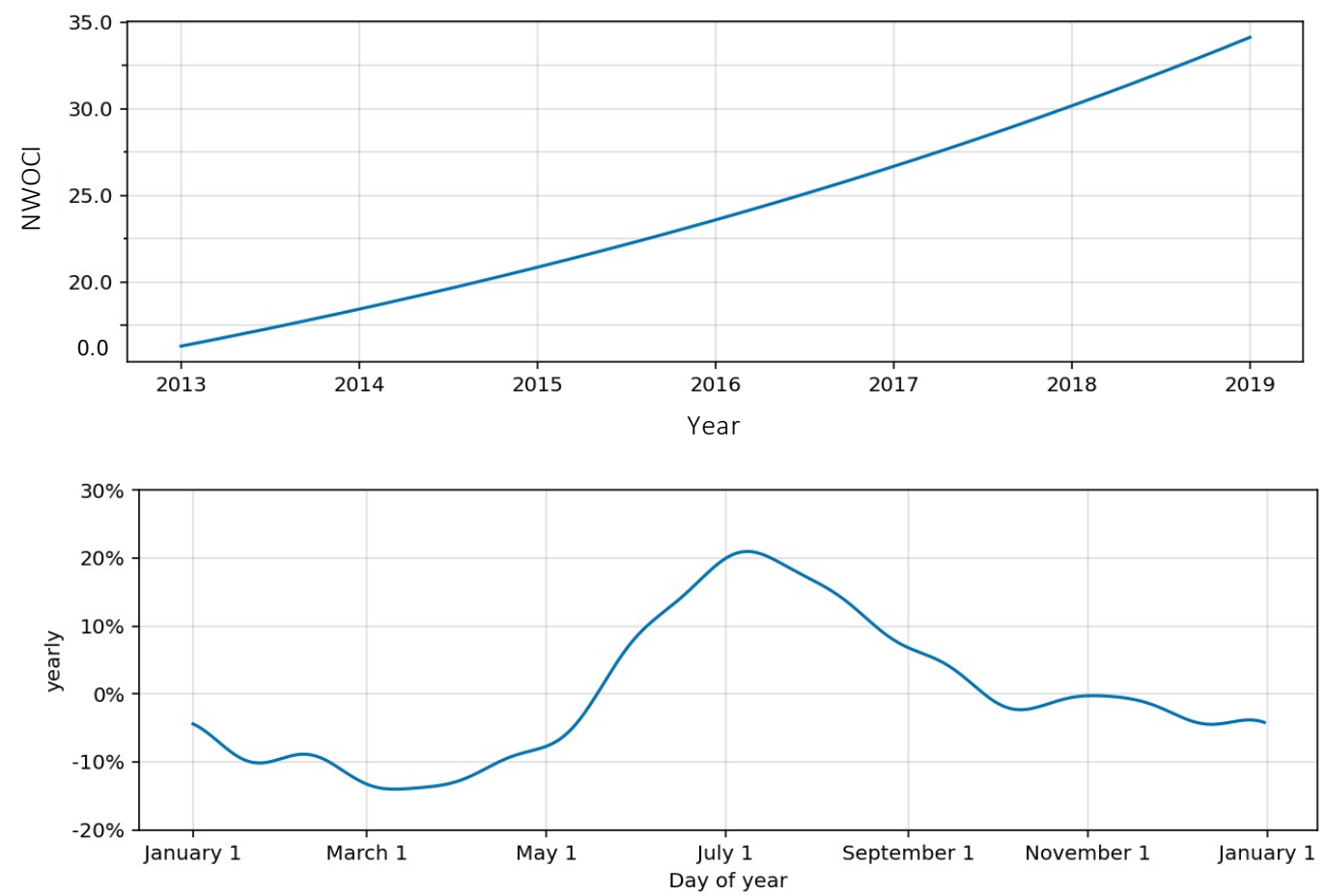

Figure 7. Top: Forecast components (trend and seasonality) with the Prophet-TPE model for the state of MA. N.B: The top panel shows the forecast trend of NWOCI based on the Prophet-TPE state level forecast. The bottom panel shows the decomposed yearly seasonal pattern of NWOCI. 
Table 3. Performance comparison of proposed Prophet-TPE model (state level) with other Prophet and SARIMA Bayesian optimized forecasting models.

\begin{tabular}{cc}
\hline & RMSE \\
\hline Prophet-TPE (Proposed Model) & 18.21 \\
Prophet-Anneal & 18.59 \\
SARIMA-TPE & 25.64 \\
SARIMA-Anneal & 22.25 \\
\hline
\end{tabular}

For the county level Prophet-BU model, we observed an improved performance in the proposed model relative to other hierarchical and Prophet forecasting models (Table 4), indicating that the proposed Prophet-BU model better captures the temporal patterns of NWOCI at the county level. As an example, the forecast result for Middlesex county using Prophet-BU model is shown in Figure 8. In the trend decomposition plot for Prophet-BU's Middlesex county forecast, we also observe a linear growth in NWOCI in the county in 2013-2018 (Figure 9). The seasonal decomposition plot also shows a summer peak in NWO in Middlesex county. The forecast and trend for all remaining 13 counties is shown in Figures A1-A13. Note that the performance of the Prophet-BU model (like any other time series model) varies depending on the amount of data available. Some counties as shown in Figures A1-A13 have very sparse data while some have more data points (e.g., Middlesex county) and, therefore, give more robust forecasts. A table of the performance of the Prophet-BU model (RMSE) across counties is given in Table A3.

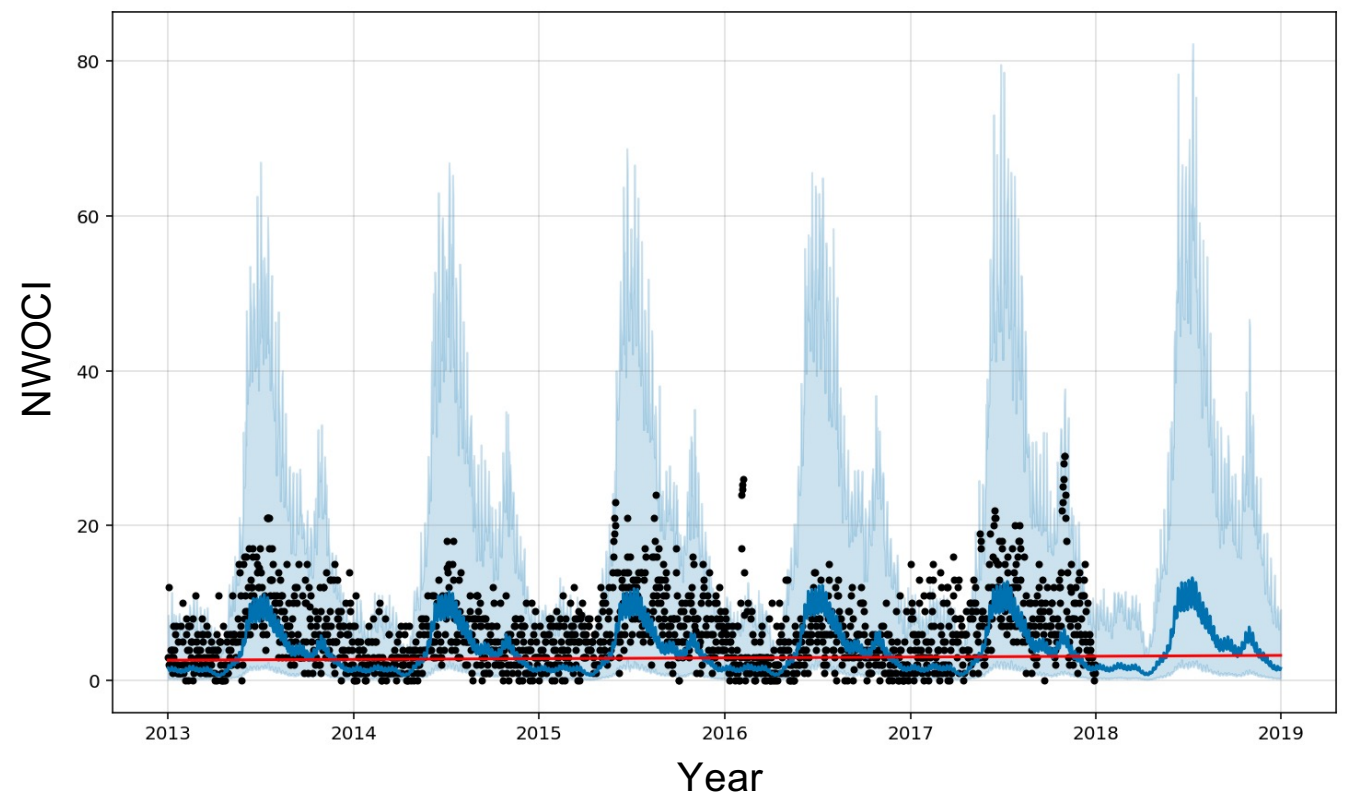

Figure 8. One year forecast result (2018 test data) from Prophet-BU Model for Middlesex county in MA (corresponding NWOCI RMSE value for Middlessex county is 59.6). N.B: The solid blue line shows the average forecast while the solid red line shows the observed trend of NWOCI and the light blue region represents the $80 \%$ confidence interval for the forecast uncertainty. Also, the scatter points represent the historical values in the training data (2013-2017). 

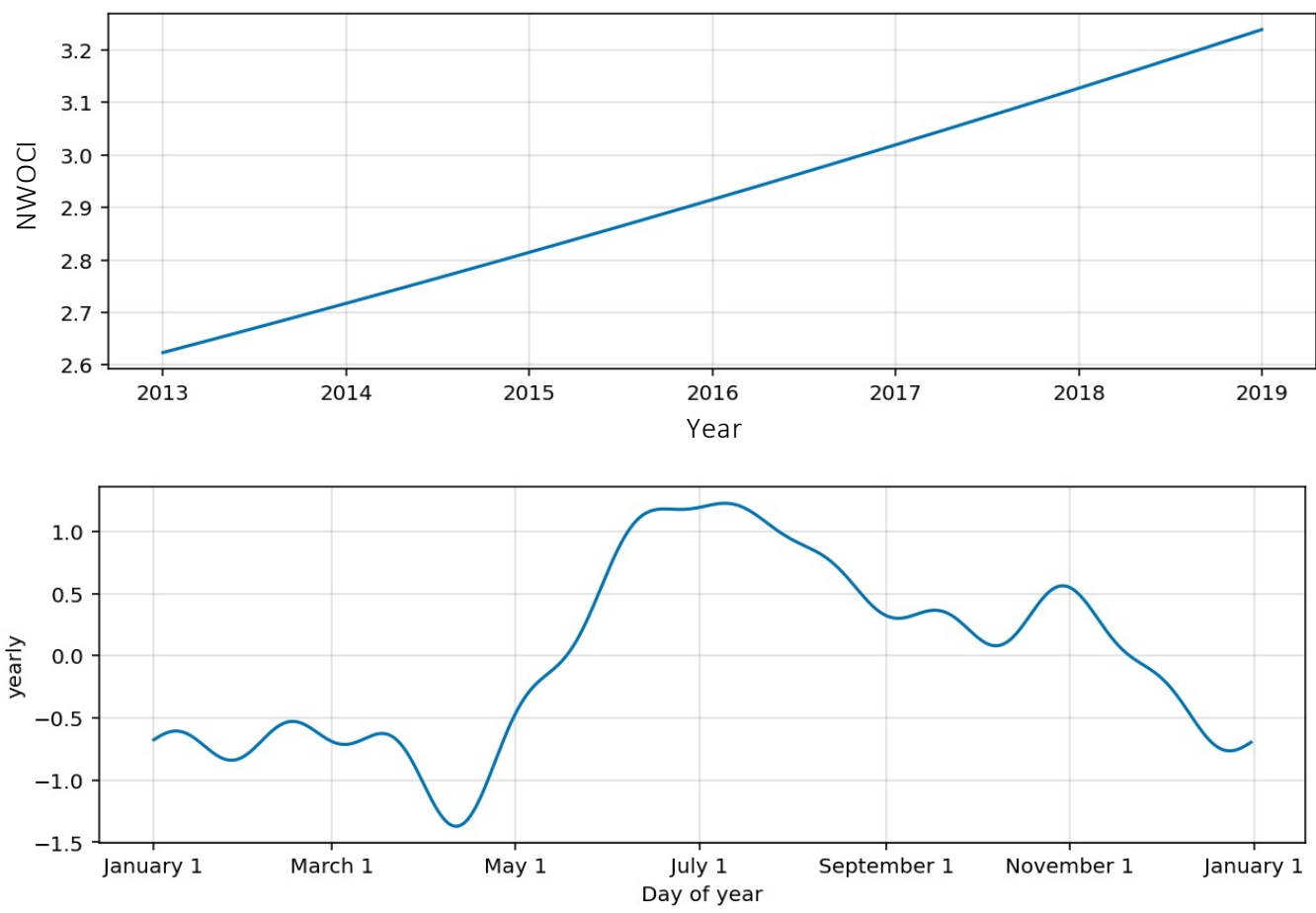

Figure 9. Forecast components (trend and seasonality) from Prophet-BU model for Middlesex county in MA. N.B: The top panel shows the forecast trend while the bottom panel shows the decomposed yearly seasonal pattern.

Table 4. Performance comparison of proposed Prophet-BU hierarchical model (county level) with other Prophet and SARIMA hierarchical forecasting models.

\begin{tabular}{cc}
\hline & RMSE \\
\hline Prophet-BU (Proposed Model) & 2.40 \\
Prophet-PHA & 2.55 \\
Prophet-AHP & 2.57 \\
Prophet-WLSS & 2.43 \\
Prophet-OLS & 2.47 \\
Prophet-WLSV & 2.59 \\
Prophet-FP & 3.81 \\
SARIMA-PHA & 3.80 \\
SARIMA-AHP & 3.80 \\
SARIMA-WLSS & 3.80 \\
SARIMA-BU & 3.81 \\
SARIMA-OLS & 3.84 \\
SARIMA-WLSV & 3.81 \\
SARIMA-FP & 3.81 \\
\hline
\end{tabular}

\section{Discussion}

As shown in Figure 10, the three main causes of NWO are: (1) failed equipment, (2) tree contact (in normal weather condition), and (3) animals.

Failed equipment accounts for 33\% of all NWOs in MA (Figure 10). This is similar to national estimates. According to a US Department of Energy study, 30\% of all non-weather related outages in the United States are a result of failed equipment [52]. Equipment failure is a common problem in electrical distribution systems and can emanate from several electrical components e.g., conductor lines, load breakers, transformers, etc. Much of these outages equipment failure can be attributed to the aging electric infrastructure [53]. The summer peak observed in the NWOCI seasonal decomposition plot (Figure 7) may be 
attributed to high demand for air conditioning in the summer. The increased electrical load and stress on the grid may ultimately lead to more electrical equipment failures.

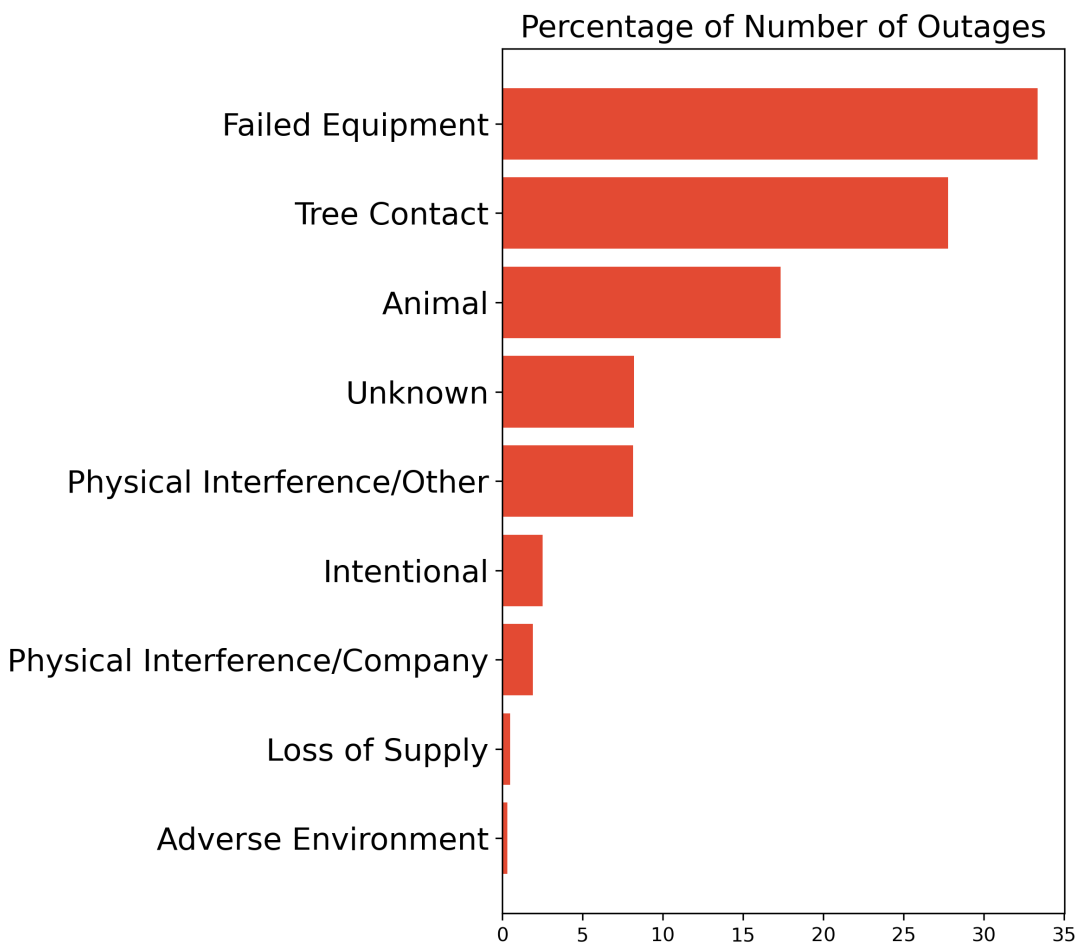

Figure 10. Bar chart showing the causes of NWO in MA based on outage data from 2013-2018 [21].

Tree contact as a result of vegetation growth is also a major problem in the electrical distribution grid. While tree contact is most prevalent in adverse weather outage events [54,55], there are tree contacts that occur even in normal weather conditions [56]. During the study period, $28 \%$ of NWOs in MA were a result of tree contact (Figure 10).

Animal-caused outages represent about $17 \%$ of NWO in MA (Figure 10). Squirrels and other animals chew electrical conductors and can cause a short-circuit leading to electric power outages $[18,57,58]$. Birds are a leading cause of overhead distribution outages in the United States as a result of their roosting, nesting, and breeding activities [59,60]. Animal interference may attribute to the observed summer peak in NWOCI (Figure 7) since there is an increased presence of migratory animals and the summer weather is more conducive for increased animal activity.

\section{Conclusions}

Unlike previous studies that focus on extreme weather outage (EWO) prediction, we highlighted the importance of capturing non-extreme weather outages (NWO), which occur more frequently and thus have substantial cumulative effect on the electrical distribution grid. We introduced a metric to quantify NWO (Non-extreme Weather Outage Count Index, NWOCI) and then proposed forecasting models to predict NWOCI at both state and county levels. We show that our proposed models outperform other Bayesian and hierarchical forecasting models in predicting NWOCI. Our proposed state-level model (Prophet-TPE) and county-level model (Prophet-BU) outperformed other models by a reduction in RMSE between $2-22 \%$ and $6-59 \%$, respectively.

The time series trend decomposition for both proposed models shows an upward trend in NWOCI indicating an increasing frequency of NWOs in MA. This reinforces the importance of developing preventive measures by stakeholders and utility managers that capture not just the EWO but also those occurring from non-extreme weather events, such as those caused by failed equipment, tree contact in normal weather conditions, and animals. These measures could include: 
1. Efforts to curb equipment failure (the leading cause of non-weather outage) by identifying weaknesses in the distribution grid and high-risk targets for predictive maintenance [52].

2. Prediction models that can forecast trends in vegetation growth and thus enable strategic tree trimming measures to be put in place.

3. Exploring the feasibility of underground power line solutions. Undergrounding electrical wires could be a solution especially for NWO which originate from animal interference. However, more research is needed on the cost-benefit tradeoff of this strategy.

4. Monitoring systems (which may be put in place by utilities) to track the rate of growth of NWOs. Such systems may reveal underlying causes of the problem and aid the development of manageable short and long term mitigation plans.

Author Contributions: Conceptualization: O.O.O.; Methodology: O.O.O.; Investigation: O.O.O.; Visualization: O.O.O.; Data Curation: O.O.O.; Formal Analysis: O.O.O.; Funding acquisition: D.A.S.; Project administration: O.O.O. and D.A.S.; Supervision: D.A.S.; Writing-original draft: O.O.O.; Writing-review \& editing: O.O.O. and D.A.S. All authors have read and agreed to the published version of the manuscript.

Funding: NSF Harnessing the Data Revolution (HDR) program, "Collaborative Research: Predictive Risk Investigation SysteM (PRISM) for Multi-layer Dynamic Interconnection Analysis" (\#1940176).

Data Availability Statement: Raw data can be found here: https:/ / github.com/Qunlexie/bayesianand-hierachical-forecasting-for-power-outages (accessed on 19 December 2021).

Conflicts of Interest: The authors declare no conflict of interest.

\section{Appendix A}

\section{Appendix A.1. Outage Data Prepossessing}

The initial data preprocessing involves data integrity and data verification procedures to address problems such as typographical errors or mismatch in the location reported for the outage. The data issues that were addressed during preprocessing include:

1. Missing Data: Missing data in the city or towns feature were filled by locating the street information (where available) on Google Maps [61]. Out of 138,153 observations, three had city/towns found in another state and two had no recorded data for city, town, or street. These observations were removed.

2. Zero values: Data points with zero customers affected or duration of outages were removed from our analysis. For example, utilities may report on failed equipment even if that failure didn't result in customers losing power (e.g., power flickers). Since there is no realized outage for these observations, they are removed from the analysis.

3. Typographical errors, extra spaces, and inconsistent names: A thorough feature inspection was carried out to ensure that the feature elements were consistent throughout. For example, typographical errors in street names and cities or towns were addressed using Google Maps [61].

\section{Appendix A.2. Computational Details}

Appendix A.2.1. Software

The complete list of software used for the study includes:

1. Kats: Kats v 0.1.0 [40] was used for the time series preprocessing to ensure a consistent time series data before developing the Bayesian and hierarchical forecasting models.

2. Prophet: Prophet v 1.0.1 [41] was used to implement the overarching Prophet forecasting model which served as a backbone for all the proposed Bayesian and hierarchical time series in this study.

3. Scikit hts: Scikit hts v 0.3.0 [51] was used to implement the hierarchical forecasting models. 
4. Hyperopt: Hyperopt v 0.2.7 [62] was used to implement the Bayesian optimization models.

Appendix A.2.2. Computational Time and Efficiency

In this study we have applied Bayesian and hierarchical models to the NWO prediction problem. While the comparison of the time complexity of these Bayesian models with other search models (random search or grid search) is beyond the scope of this article, this has been extensively studied to buttress the efficiency and computational efficiency of Bayesian models $[48,62,63]$. The Bayesian optimization methods explored in this study are known to be among the most efficient in terms of obtaining the optimal set of hyperparameters by intelligently using information on the performance of hyperparameters from the training process.

Appendix A.3. Tables

Table A1. Hyperparameter search domain for the Prophet and SARIMA models.

\begin{tabular}{|c|c|}
\hline Model & Search Domain \\
\hline Prophet & $\begin{array}{c}\text { 'changepoint_prior_scale': [0.0001, 0.0005, 0.001, 0.005], } \\
\text { 'changepoint_range': [0.8, 0.85, 0.9, 0.95], } \\
\text { 'seasonality_mode': ['additive', 'multiplicative'], } \\
\text { 'seasonality_prior_scale': [0.01, 0.1, 1.0, 10.0], } \\
\text { 'yearly_seasonality': [True, False] }\end{array}$ \\
\hline SARIMA & 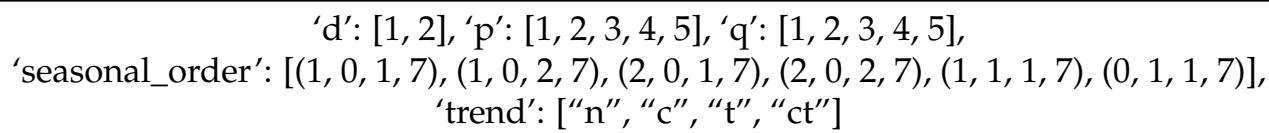 \\
\hline
\end{tabular}

Table A2. Hyperparameter results for state-level NWOCI forecast showing the optimal hyperparameter values for each of the four Bayesian Prophet and SARIMA models.

\begin{tabular}{|c|c|}
\hline Model & Hyperparameters \\
\hline Prophet-TPE & $\begin{array}{l}\text { 'changepoint_prior_scale': } 0.0001, \\
\text { 'changepoint_range': 0.9, } \\
\text { 'seasonality_mode': 'additive', } \\
\text { 'seasonality_prior_scale': } 10.0, \\
\text { 'yearly_seasonality': True }\end{array}$ \\
\hline Prophet-Anneal & $\begin{array}{c}\text { 'changepoint_prior_scale': } 0.005, \\
\text { 'changepoint_range': } 0.85, \\
\text { 'seasonality_mode': 'multiplicative', } \\
\text { 'seasonality_prior_scale': } 0.1, \\
\text { 'yearly_seasonality': True }\end{array}$ \\
\hline SARIMA-TPE & $\begin{array}{c}\text { 'd': } 1,{ }^{\prime} \mathrm{p}^{\prime}: 1,{ }^{\prime} \mathrm{q} \text { ': } 3, \\
\text { 'seasonal_order': }(2,0,2,7), \text { 'trend': ' } \mathrm{t} \text { ' }\end{array}$ \\
\hline SARIMA-Anneal & $\begin{array}{c}\text { 'd': } 1, \text { 'p': } 1, \text { ' } \mathrm{q}^{\prime}: 5, \\
\text { 'seasonal_order': }(1,0,1,7), \text { 'trend': 't' }\end{array}$ \\
\hline
\end{tabular}


Table A3. Performance of the Proposed Prophet BU model for each county in MA.

\begin{tabular}{cc}
\hline & RMSE \\
\hline Barnstable & 3.63 \\
Berkshire & 2.44 \\
Bristol & 14.10 \\
Dukes & 2.44 \\
Berkshire & 0.41 \\
Essex & 3.18 \\
Franklin & 1.98 \\
Hampden & 3.97 \\
Hampshire & 1.57 \\
Middlesex & 59.65 \\
Nantucket & 0.22 \\
Norfolk & 3.86 \\
Plymouth & 4.04 \\
Suffolk & 5.56 \\
Worcester & 4.65 \\
\hline
\end{tabular}

Appendix A.4. Figures

\section{BARNSTABLE}
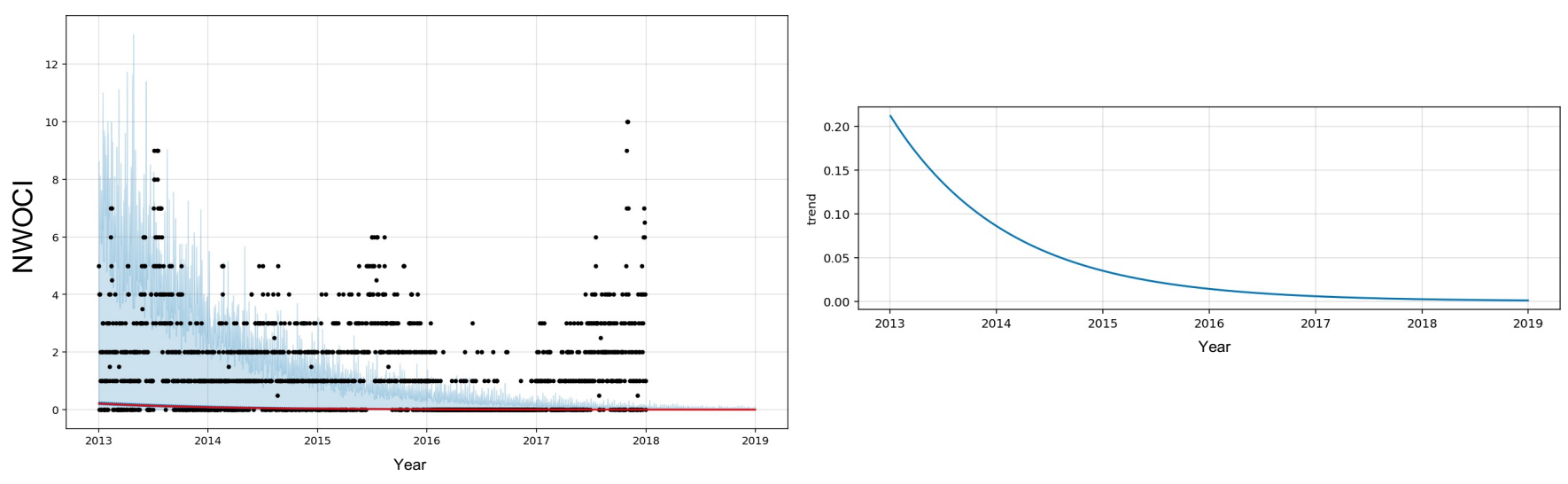

Figure A1. Left: One year forecast result (2018 test data) from Prophet-BU Model for Barnstable county in MA. N.B: The solid blue line shows the average forecast while the solid red line shows the observed trend of NWOCI and the light blue region represents the $80 \%$ confidence interval for the forecast uncertainty. Also, the scatter points represent the historical values in the training data (2013-2017). Right: Trend decomposition from Prophet-BU Model for Barnstable county in MA. 


\section{BERKSHIRE}
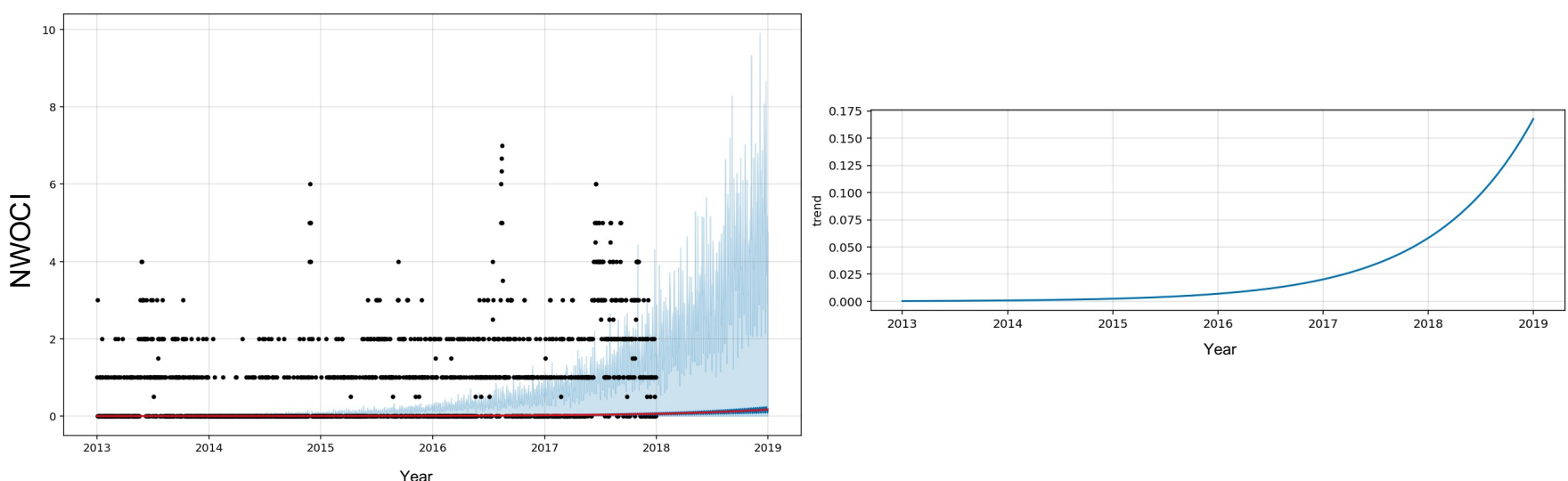

Figure A2. Left: One year forecast (2018 test data) from Prophet-BU Model for Berkshire county in MA. N.B: The solid blue line shows the average forecast while the solid red line shows the observed trend of NWOCI and the light blue region represents the $80 \%$ confidence interval for the forecast uncertainty. Also, the scatter points represent the historical values in the training data (2013-2017). Right: Trend decomposition from Prophet-BU Model for Berkshire county in MA.

\section{BRISTOL}
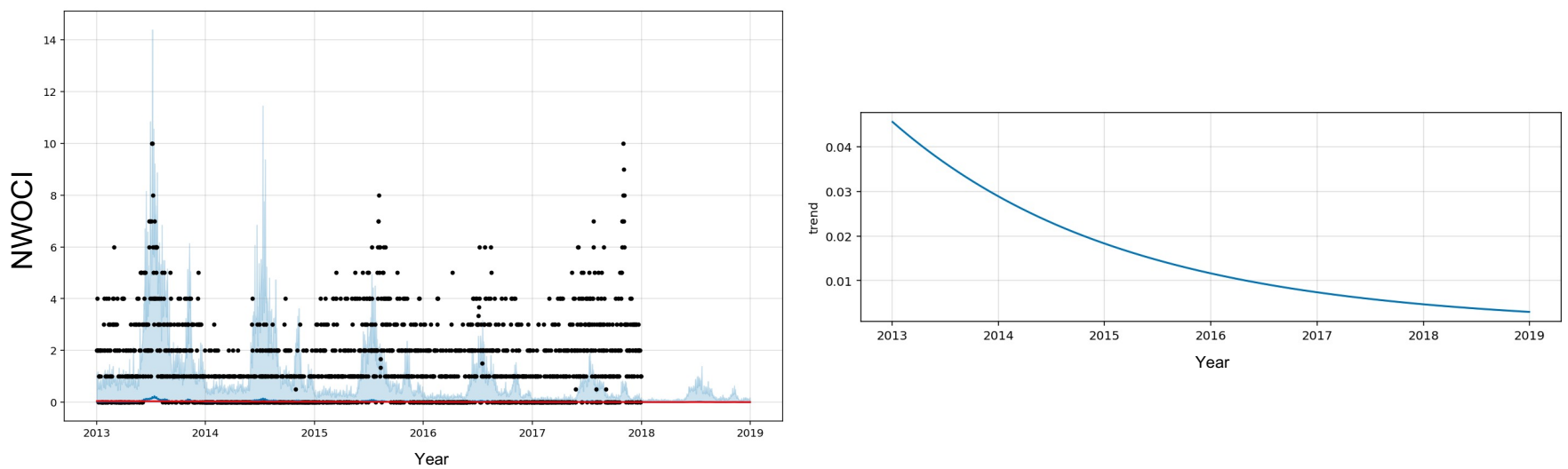

Figure A3. Left: One year forecast (2018 test data) from Prophet-BU Model for Bristol county in MA. N.B: The solid blue line shows the average forecast while the solid red line shows the observed trend of NWOCI and the light blue region represents the $80 \%$ confidence interval for the forecast uncertainty. Also, the scatter points represent the historical values in the training data (2013-2017). Right: Trend decomposition from Prophet-BU Model for Bristol county in MA. 


\section{DUKES}
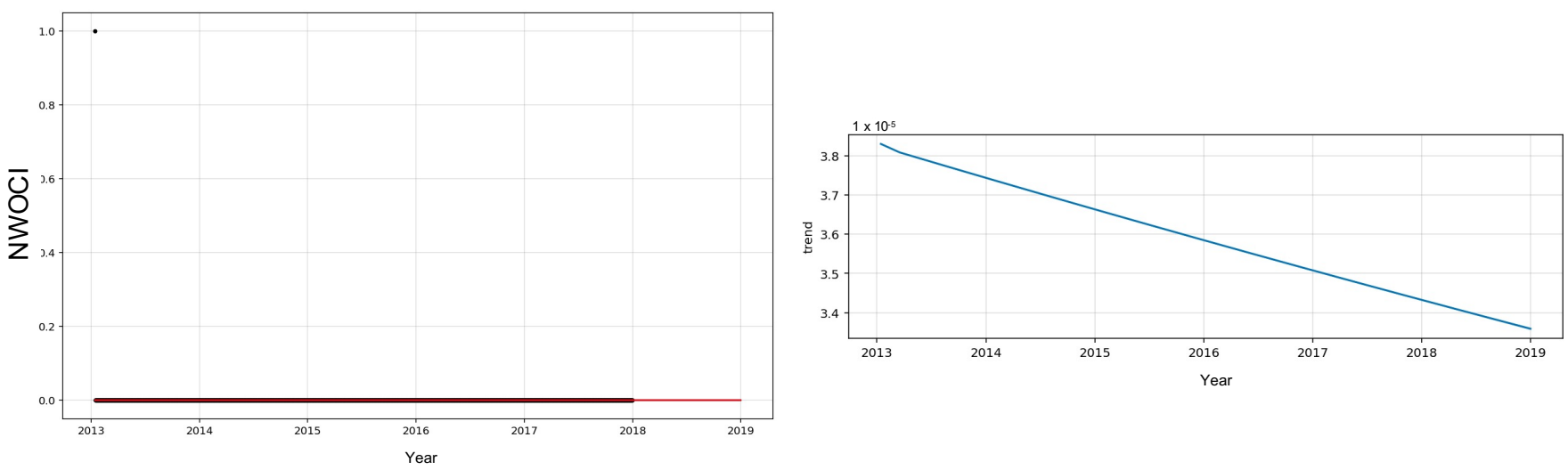

Figure A4. Left: One year forecast (2018 test data) from Prophet-BU Model for Dukes county in MA. N.B: The solid blue line shows the average forecast while the solid red line shows the observed trend of NWOCI and the light blue region represents the $80 \%$ confidence interval for the forecast uncertainty. Also, the scatter points represent the historical values in the training data (2013-2017). Right: Trend decomposition from Prophet-BU Model for Dukes county in MA.

\section{ESSEX}
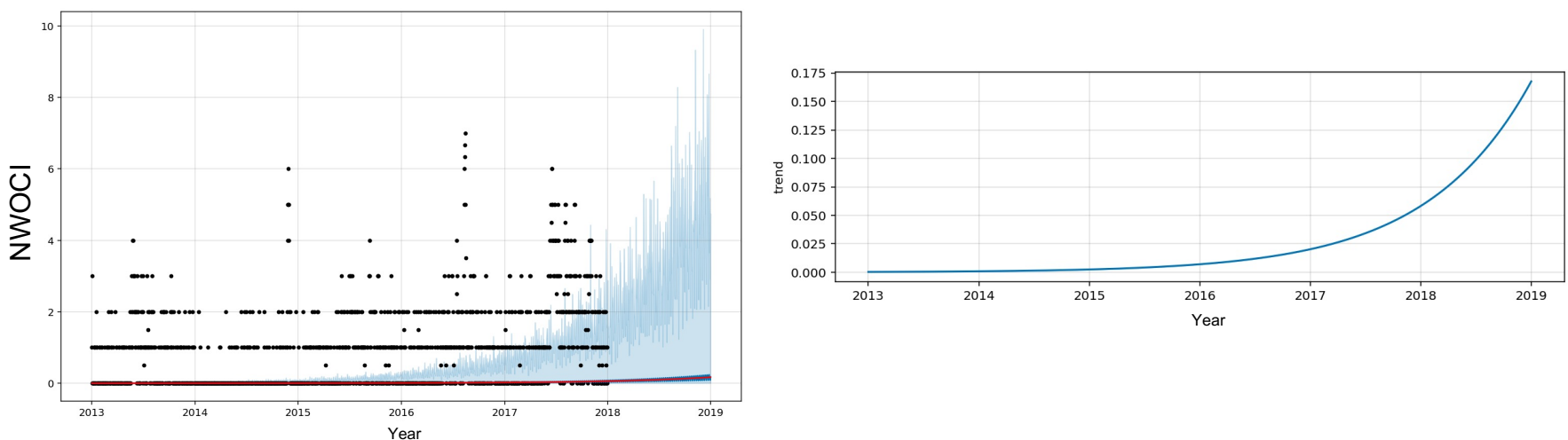

Figure A5. Left: One year forecast (2018 test data) from Prophet-BU Model for Essex county in MA. N.B: The solid blue line shows the average forecast while the solid red line shows the observed trend of NWOCI and the light blue region represents the $80 \%$ confidence interval for the forecast uncertainty. Also, the scatter points represent the historical values in the training data (2013-2017). Right: Trend decomposition from Prophet-BU Model for Essex county in MA. 


\section{FRANKLIN}
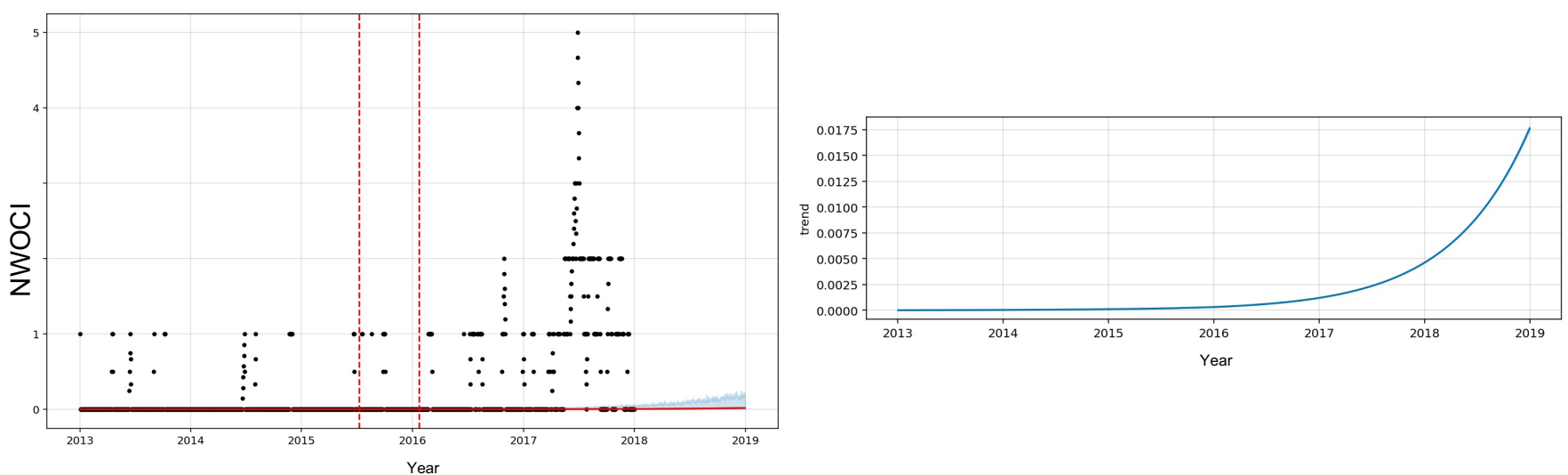

Figure A6. Left: One year forecast (2018 test data) from Prophet-BU Model for Franklin county in MA. N.B: The solid blue line shows the average forecast while the solid red line shows the observed trend of NWOCI and the light blue region represents the $80 \%$ confidence interval for the forecast uncertainty. Also, the scatter points represent the historical values in the training data (2013-2017). Right: Corresponding trend decomposition from Prophet-BU Model.

\section{HAMPSHIRE}
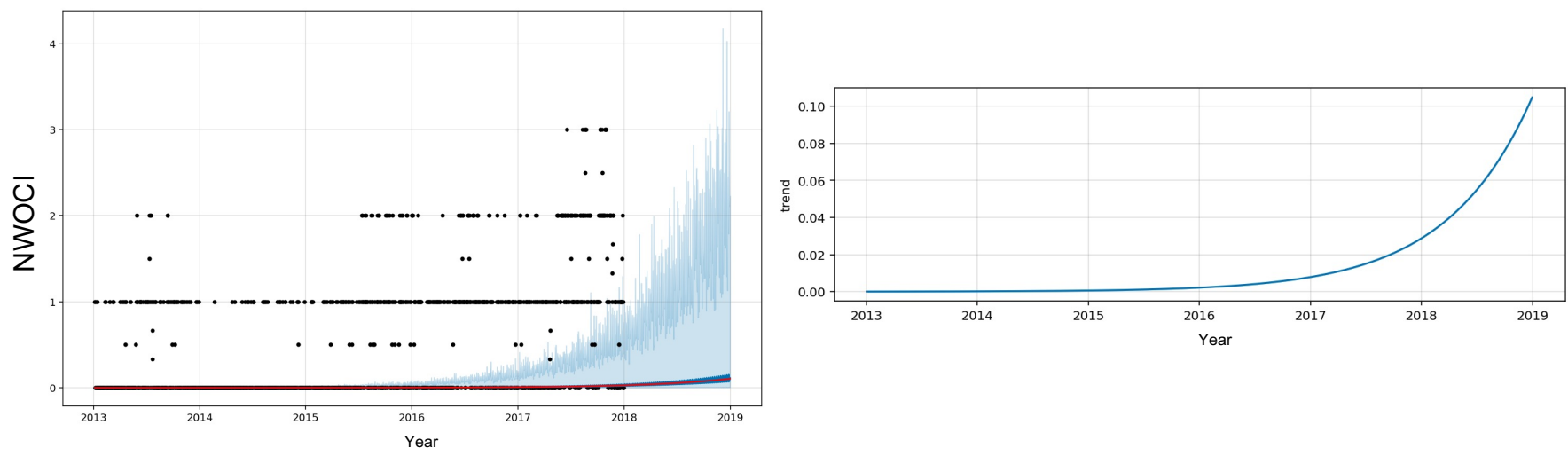

Figure A7. Left: One year forecast (2018 test data) from Prophet-BU Model for Hamphsire county in MA. N.B: The solid blue line shows the average forecast while the solid red line shows the observed trend of NWOCI and the light blue region represents the $80 \%$ confidence interval for the forecast uncertainty. Also, the scatter points represent the historical values in the training data (2013-2017). Right: Corresponding trend decomposition from Prophet-BU Model. 


\section{HAMPDEN}
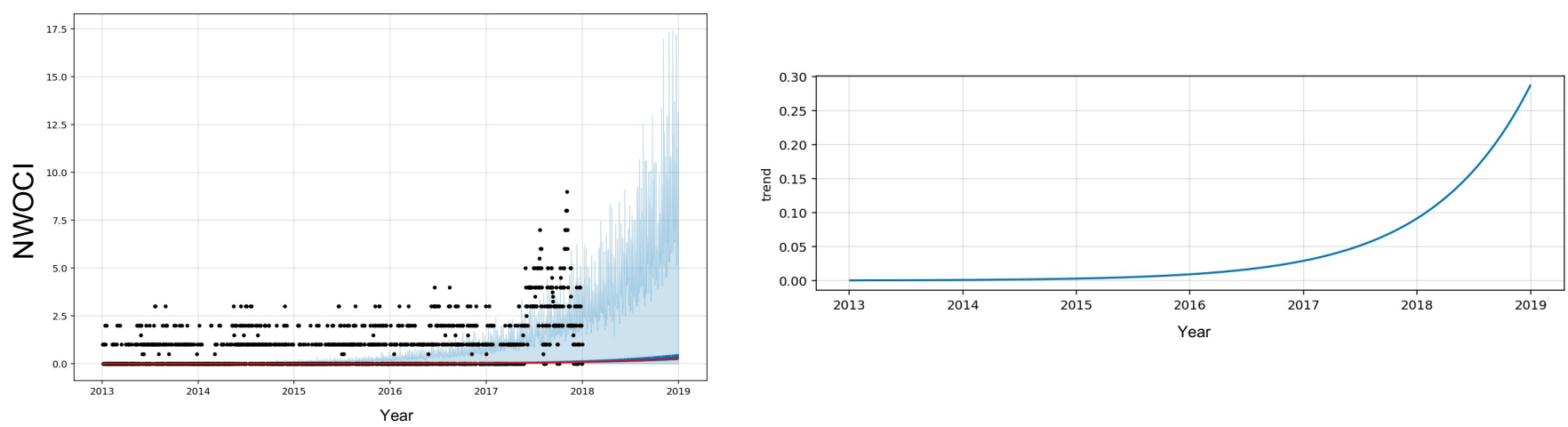

Figure A8. Left: One year forecast (2018 test data) from Prophet-BU Model for Hampden county in MA. N.B: The solid blue line shows the average forecast while the solid red line shows the observed trend of NWOCI and the light blue region represents the $80 \%$ confidence interval for the forecast uncertainty. Also, the scatter points represent the historical values in the training data (2013-2017). Right: Corresponding trend decomposition from Prophet-BU Model.

\section{NANTUCKET}
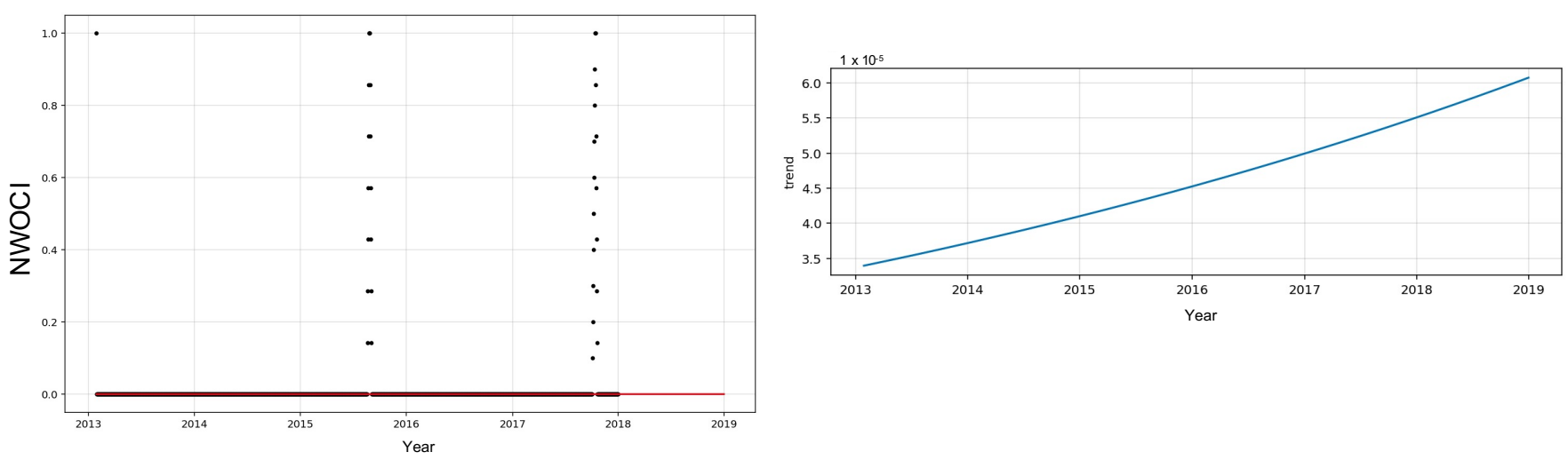

Figure A9. Left: One year forecast (2018 test data) from Prophet-BU Model for Nantucket county in MA. N.B: The solid blue line shows the average forecast while the solid red line shows the observed trend of NWOCI and the light blue region represents the $80 \%$ confidence interval for the forecast uncertainty. Also, the scatter points represent the historical values in the training data (2013-2017). Right: Corresponding trend decomposition from Prophet-BU Model. 


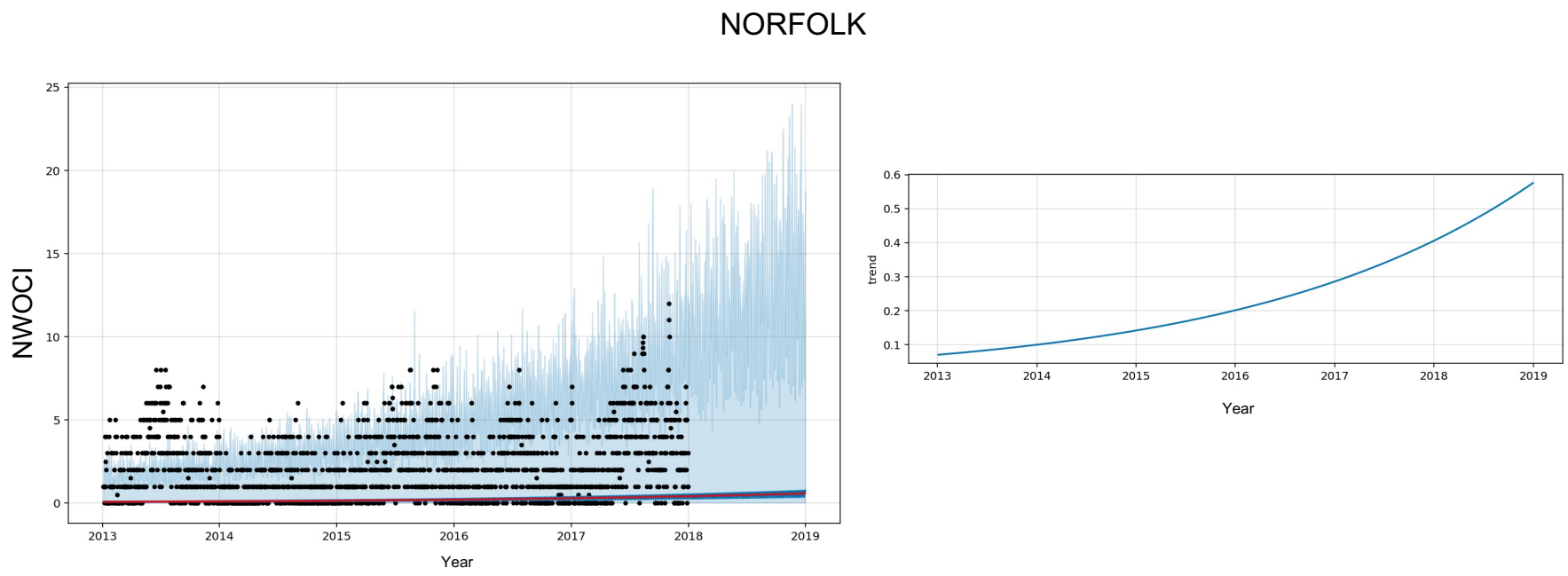

Figure A10. Left: One year forecast (2018 test data) from Prophet-BU Model for Norfolk county in MA. N.B: The solid blue line shows the average forecast while the solid red line shows the observed trend of NWOCI and the light blue region represents the $80 \%$ confidence interval for the forecast uncertainty. Also, the scatter points represent the historical values in the training data (2013-2017). Right: Corresponding trend decomposition from Prophet-BU Model.

\section{PLYMOUTH}
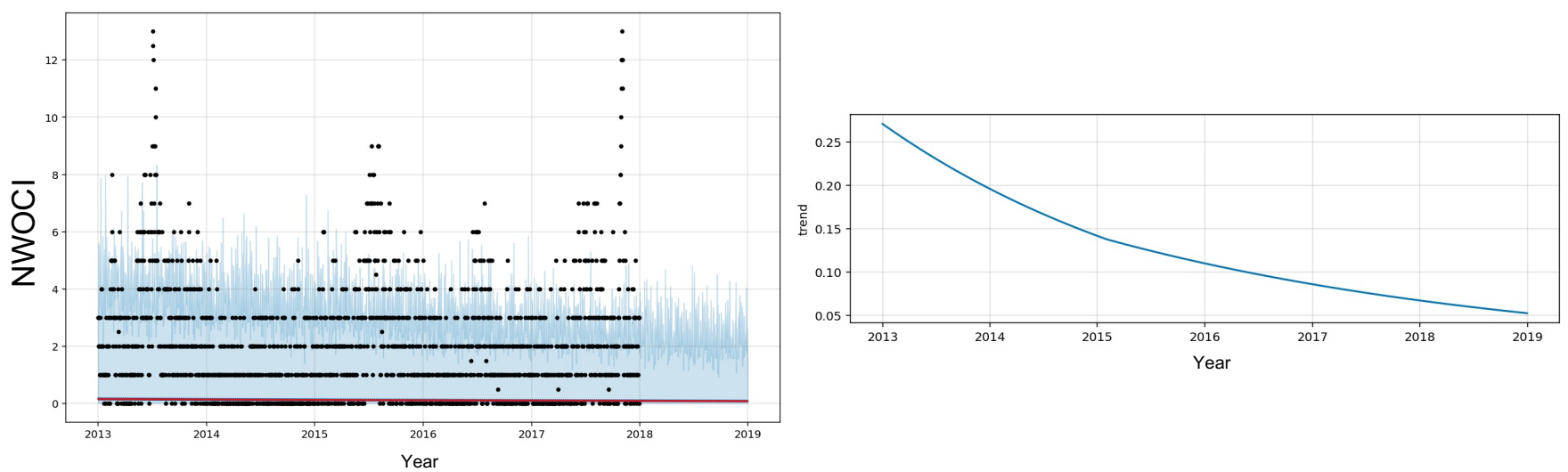

Figure A11. Left: One year forecast (2018 test data) from Prophet-BU Model for Franklin county in MA. N.B: The solid blue line shows the average forecast while the solid red line shows the observed trend of NWOCI and the light blue represents region represents the $80 \%$ confidence interval for the forecast uncertainty. Also, the scatter points represent the historical values in the training data (2013-2017). Right: Corresponding trend decomposition from Prophet-BU Model. 


\section{SUFFOLK}
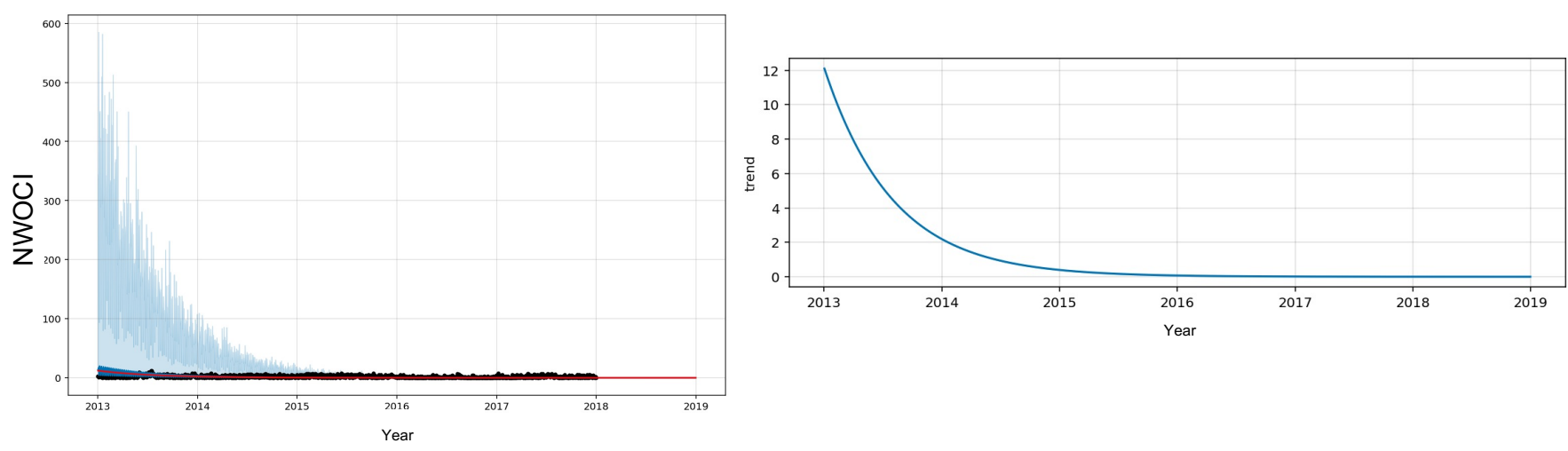

Figure A12. Left: One year forecast (2018 test data) from Prophet-BU Model for Suffolk county in MA. N.B: The solid blue line shows the average forecast while the solid red line shows the observed trend of NWOCI and the light blue region represents the $80 \%$ confidence interval for the forecast uncertainty. Also, the scatter points represent the historical values in the training data (2013-2017). Right: Corresponding trend decomposition from Prophet-BU Model.

WORCESTER
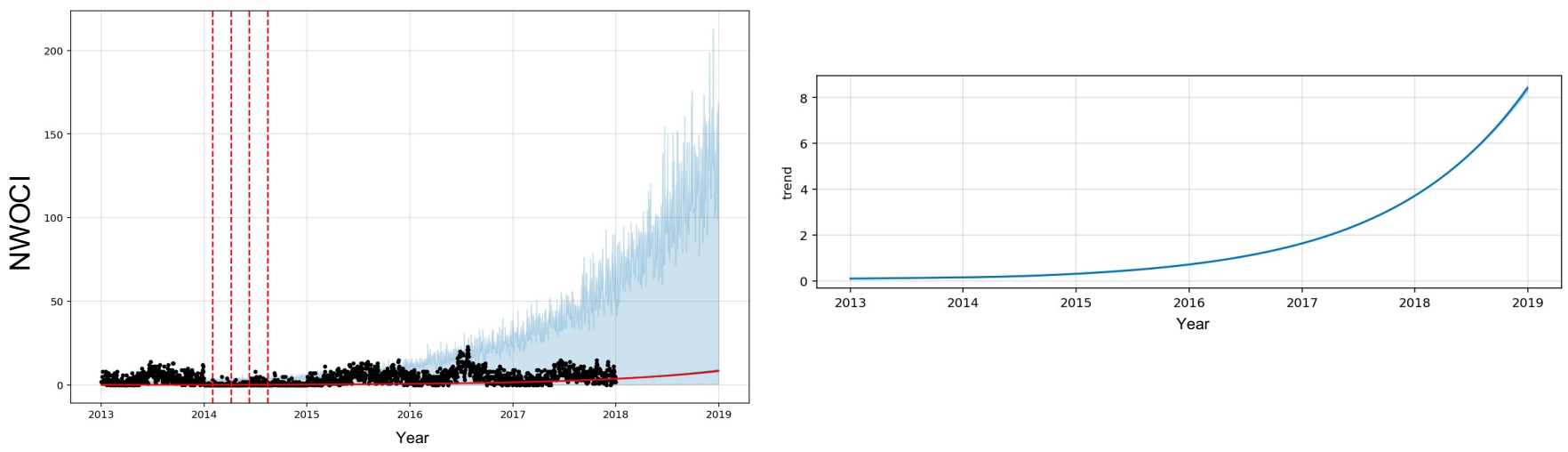

Figure A13. Left: One year forecast (2018 test data) from Prophet-BU Model for Worcester county in MA. N.B: The solid blue line shows the average forecast while the solid red line shows the observed trend of NWOCI and the light blue region represents the $80 \%$ confidence interval for the forecast uncertainty. Also, the scatter points represent the historical values in the training data (2013-2017). The red dotted lines show the time series change point delineated by the prophet model. Right: Corresponding trend decomposition from Prophet-BU Model.

\section{References}

1. Conti, J.P. The day the samba stopped [power blackouts]. Eng. Technol. 2010, 5, 46-47. [CrossRef]

2. Koc, Y.; Verma, T.; Araujo, N.A.; Warnier, M. MATCASC: A tool to analyse cascading line outages in power grids. In Proceedings of the 2013 IEEE International Workshop on Intelligent Energy Systems, IWIES 2013, Vienna, Austria, 14 November 2013; pp. 143-148. [CrossRef]

3. Burpee, D.; Dabaghi, H.; Jackson, L.; Kwamena, F.; Richter, J.; Rusnov, T.; Friedman, K.; Mansueti, L.; Meyer, D. U.S.-Canada Power System Outage Task Force: Final Report on the Implementation of Task Force Recommendations. 2006. Available online: https: / / www.energy.gov/oe/downloads/us-canada-power-system-outage-task-force-final-report-implementationtask-force (accessed on 21 December 2021).

4. Che-Castaldo, J.; Cousin, R.; Daryanto, S.; Deng, G.; Feng, M.E.; Gupta, R.K.; Hong, D.; McGranaghan, R.M.; Owolabi, O.O.; Qu, T.; et al. Critical Risk Indicators (CRIs) for the electric power grid: A survey and discussion of interconnected effects. Environ. Syst. Decis. 2021, 41, 594-615. [CrossRef] [PubMed]

5. Eeeten, M.V.; Nieuwenhuijs, A.; Luiijf, E.; Klaver, M.; Cruz, E. The state and the threat of cascading failure across critical infrastructures: The implications of empirical evidence from media incident reports. Public Adm. 2011, 89, 381-400. [CrossRef] 
6. Schneider, C.M.; Yazdani, N.; Araújo, N.A.M.; Havlin, S.; Herrmann, H.J. Towards designing robust coupled networks. Sci. Rep. 2013, 3, 1969. [CrossRef]

7. Buldyrev, S.V.; Parshani, R.; Paul, G.; Stanley, H.E.; Havlin, S. Catastrophic cascade of failures in interdependent networks. Nature 2010, 464, 1025-1028. [CrossRef] [PubMed]

8. Kosa, K.M.; Cates, S.C.; Karns, S.; Godwin, S.L.; Coppings, R.J. Are Older Adults Prepared to Ensure Food Safety During Extended Power Outages and Other Emergencies? Findings from a National Survey. Educ. Gerontol. 2012, 38, 763-775. [CrossRef]

9. Kosa, K.M.; Cates, S.C.; Godwin, S.L.; Coppings, R.J.; Speller-Henderson, L. Most Americans are Not Prepared to Ensure Food Safety during Power Outages and Other Emergencies. Food Prot. Trends 2011, 31, 428-436.

10. Jan, S.; Lurie, N. Disaster Resilience and People with Functional Needs. New Engl. J. Med. 2012, 367, 2272-2273. [CrossRef]

11. Wear, J.O. Is Your Hospital Ready for a Natural or Man-Made Disaster. IFMBE Proc. 2011, 37, 699-702.

12. Alshawish, A.; Meer, H.U. Risk-based decision-support for vulnerability remediation in electric power networks. In Proceedings of the 10th ACM International Conference on Future Energy Systems, e-Energy 2019, Phoenix, AZ, USA, 25-28 June 2019; pp. 378-380. [CrossRef]

13. Alshawish, A.; de Meer, H. Risk mitigation in electric power systems: Where to start? Energy Inform. 2019, 2, 34. [CrossRef]

14. Alpay, B.A.; Wanik, D.; Watson, P.; Cerrai, D.; Liang, G.; Anagnostou, E. Dynamic Modeling of Power Outages Caused by Thunderstorms. Forecasting 2020, 2, 151-162. [CrossRef]

15. Wanik, D.W.; Anagnostou, E.N.; Astitha, M.; Hartman, B.M.; Lackmann, G.M.; Yang, J.; Cerrai, D.; He, J.; Frediani, M.E.B. A Case Study on Power Outage Impacts from Future Hurricane Sandy Scenarios. J. Appl. Meteorol. Climatol. 2018, 57, 51-79. [CrossRef]

16. Wang, Y.; Chen, C.; Wang, J.; Baldick, R. Research on Resilience of Power Systems Under Natural Disasters-A Review. IEEE Trans. Power Syst. 2016, 31, 1604-1613. [CrossRef]

17. Hines, P.; Apt, J.; Talukdar, S. Trends in the history of large blackouts in the United States. In Proceedings of the 2008 IEEE Power and Energy Society General Meeting-Conversion and Delivery of Electrical Energy in the 21st Century, Pittsburgh, PA, USA, 20-24 July 2008; pp. 1-8. [CrossRef]

18. Doostan, M.; Chowdhury, B. Statistical Analysis of Animal-Related Outages in Power Distribution Systems-A Case Study. In Proceedings of the 2019 IEEE Power \& Energy Society General Meeting (PESGM), Atlanta, GA, USA, 4-8 August 2019; pp. 1-5. [CrossRef]

19. Sahai, S.; Pahwa, A. A probabilistic approach for animal-caused outages in overhead distribution systems. In Proceedings of the 2006 International Conference on Probabilistic Methods Applied to Power Systems, Stockholm, Sweden, 11-15 June 2006. [CrossRef]

20. Gui, M.; Pahwa, A.; Das, S. Analysis of animal-related outages in overhead distribution systems with wavelet decomposition and immune systems-based neural networks. IEEE Trans. Power Syst. 2009, 24, 1765-1771. [CrossRef]

21. Department of Public Utilities: Energy and Environmental Affairs. Fileroom. 2013. Available online: https://eeaonline.eea.state ma.us/DPU/Fileroom/dockets/bynumber (accessed on 4 May 2020).

22. Cerrai, D.; Wanik, D.W.; Bhuiyan, A.E.; Zhang, X.; Yang, J.; Frediani, M.E.B.; Anagnostou, E.N. Predicting Storm Outages Through New Representations of Weather and Vegetation. IEEE Access 2019, 7, 29639-29654. [CrossRef]

23. Zhai, C.; Chen, T.Y.-J.; White, A.G.; Guikema, S.D. Power outage prediction for natural hazards using synthetic power distribution systems. Reliab. Eng. Syst. Saf. 2021, 208, 107348. [CrossRef]

24. Tervo, R.; Láng, I.; Jung, A.; Mäkelä, A. Predicting power outages caused by extratropical storms. Nat. Hazards Earth Syst. Sci. 2021, 21, 607-627. [CrossRef]

25. Guikema, S.D. Natural disaster risk analysis for critical infrastructure systems: An approach based on statistical learning theory. Reliab. Eng. Syst. Saf. 2009, 94, 855-860. [CrossRef]

26. Guikema, S.D.; Quiring, S.M.; Han, S.R. Prestorm Estimation of Hurricane Damage to Electric Power Distribution Systems. Risk Anal. 2010, 30, 1744-1752. [CrossRef]

27. Guikema, S.D.; Nateghi, R.; Quiring, S.M.; Staid, A.; Reilly, A.C.; Gao, M. Predicting Hurricane Power Outages to Support Storm Response Planning. IEEE Access 2014, 2, 1364-1373. [CrossRef]

28. Wang, G.; Xu, T.; Tang, T.; Yuan, T.; Wang, H. A Bayesian network model for prediction of weather-related failures in railway turnout systems. Expert Syst. Appl. 2017, 69, 247-256. [CrossRef]

29. Nateghi, R.; Guikema, S.D.; Quiring, S.M. Comparison and Validation of Statistical Methods for Predicting Power Outage Durations in the Event of Hurricanes. Risk Anal. 2011, 31, 1897-1906. [CrossRef] [PubMed]

30. Nateghi, R.; Guikema, S.; Quiring, S.M. Power Outage Estimation for Tropical Cyclones: Improved Accuracy with Simpler Models. Risk Anal. 2014, 34, 1069-1078. [CrossRef] [PubMed]

31. Han, S.R.; Guikema, S.D.; Quiring, S.M. Improving the Predictive Accuracy of Hurricane Power Outage Forecasts Using Generalized Additive Models. Risk Anal. 2009, 29, 1443-1453. [CrossRef]

32. Yang, F.; Wanik, D.W.; Cerrai, D.; Bhuiyan, M.A.E.; Anagnostou, E.N. Quantifying Uncertainty in Machine Learning-Based Power Outage Prediction Model Training: A Tool for Sustainable Storm Restoration. Sustainability 2020, 12, 1525. [CrossRef]

33. Yue, M.; Toto, T.; Jensen, M.P.; Giangrande, S.E.; Lofaro, R. A Bayesian Approach-Based Outage Prediction in Electric Utility Systems Using Radar Measurement Data. IEEE Trans. Smart Grid 2018, 9, 6149-6159. [CrossRef]

34. He, J.; Wanik, D.W.; Hartman, B.M.; Anagnostou, E.N.; Astitha, M.; Frediani, M.E.B. Nonparametric Tree-Based Predictive Modeling of Storm Outages on an Electric Distribution Network. Risk Anal. 2017, 37, 441-458. [CrossRef] 
35. Zhu, D.; Cheng, D.; Broadwater, R.P.; Scirbona, C. Storm modeling for prediction of power distribution system outages. Electr. Power Syst. Res. 2007, 77, 973-979. [CrossRef]

36. Lubkeman, D.; Julian, D. Large scale storm outage management. In Proceedings of the IEEE Power Engineering Society General Meeting, Denver, CO, USA, 6-10 June 2004; Volume 2, pp. 16-22. [CrossRef]

37. Eskandarpour, R.; Khodaei, A.; Arab, A. Improving power grid resilience through predictive outage estimation. In Proceedings of the 2017 North American Power Symposium (NAPS), Morgantown, WV, USA, 17-19 September 2017.

38. Feng, M.L.E.; Owolabi, O.O.; Schafer, T.L.J.; Sengupta, S.; Wang, L.; Matteson, D.S.; Che-Castaldo, J.P.; Sunter, D.A. Analysis of animal-related electric outages using species distribution models and community science data. arXiv 2021, arXiv:2112.12791.

39. Doostan, M.; Sohrabi, R.; Chowdhury, B. A data-driven approach for predicting vegetation-related outages in power distribution systems. Int. Trans. Electr. Energy Syst. 2020, 30, e12154. [CrossRef]

40. Facebook. Kats I One Stop Shop for Time Series. 2021. Available online:https:/ facebookresearch.github.io/Kats/ (accessed on 14 September 2021).

41. Facebook. Prophet I Forecasting at Scale. 2021. Available online: https:// facebook.github.io/prophet/ (accessed on 14 September 2021).

42. Shahriari, B.; Swersky, K.; Wang, Z.; Adams, R.P.; Freitas, N.D. Taking the Human Out of the Loop: A Review of Bayesian Optimization. Proc. IEEE 2016, 104, 148-175. [CrossRef]

43. Hyndman, R.; Athanasopoulos, G. Forecasting: Principles and Practice; OTexts: Heathmont, Australia, 2014.

44. National Oceanic and Atmospheric Administration. Climate Data Online. 2021. Available online: https://www.ncdc.noaa.gov/ cdo-web/datasets\#LCD (accessed on 21 December 2021).

45. Britannica. Meteorology: Gust. 2021. Available online: https://www.britannica.com/science/gust (accessed on 21 December 2021).

46. National Weather Service US Department of Commerce, NOAA. Wind Threat Defined. Available online: https://www.weather. gov/mlb/wind_threat (accessed on 21 December 2021).

47. Taylor, S.J.; Letham, B. Forecasting at Scale. PeerJ Prepr. 2017, 5, e3190v2. [CrossRef]

48. Bergstra, J.; Bardenet, R.; Bengio, Y.; Kégl, B. Algorithms for Hyper-Parameter Optimization. In Proceedings of the 25th Annual Conference on Neural Information Processing Systems, Granada, Spain, 12-15 December 2011.

49. Dewancker, I.; Mccourt, M.; Clark, S. Bayesian Optimization Primer. 2017. Available online: https://static.sigopt.com/b/20a144 d208ef255d3b981ce419667ec25d8412e2/static/pdf/SigOpt_Bayesian_Optimization_Primer.pdf (accessed on 21 December 2021).

50. Hyndman, R.J.; Ahmed, R.A.; Athanasopoulos, G.; Shang, H.L. Optimal combination forecasts for hierarchical time series. Comput. Stat. Data Anal. 2011, 55, 2579-2589. [CrossRef]

51. Scikit Hts. Scikit-Hts-Hts 0.5.12 Documentation. 2021. Available online: https://scikit-hts.readthedocs.io/en/latest/readme. html\#credits (accessed on 21 December 2021).

52. T\&D World. Failure: When and Where? 2015. Available online: https://www.tdworld.com/grid-innovations/smart-grid/ article/20965853/failure-when-and-where (accessed on 21 December 2021).

53. ASCE. Infrastructure Report Card. 2021. Available online: https://infrastructurereportcard.org/cat-item/energy/ (accessed on 21 December 2021).

54. Campbell, R.J. CRS Report for Congress Weather-Related Power Outages and Electric System Resiliency. 2012. Available online: https://sgp.fas.org/crs/misc/R42696.pdf (accessed on 21 December 2021).

55. Hartling, S.; Sagan, V.; Maimaitijiang, M.; Dannevik, W.; Pasken, R. Estimating tree-related power outages for regional utility network using airborne LiDAR data and spatial statistics. Int. J. Appl. Earth Obs. Geoinf. 2021, 100, 102330. [CrossRef]

56. Duquesne Light Company. Causes of Power Outages. 2021. Available online: https://www.duquesnelight.com/outages-safety/ restoring-power/causes-of-power-outages (accessed on 14 November 2021).

57. Hamilton, J.C.; Johnson, R.J.; Case, R.M.; Riley, M.W.; Chris, J. Assessment of Squirrel-Caused Power Outages. ASTM Spec. Tech. Publ. 1989, 1055, 34-40.

58. Chow, M.Y. Analysis and Prevention of Animal-Caused Faults in Power Distribution Systems. IEEE Trans. Power Deliv. 1995, 10, 995-1001. [CrossRef]

59. Burgio, K.; Rubega, M.; Sustaita, D. Nest-building behavior of Monk Parakeets and insights into potential mechanisms for reducing damage to utility poles. PeerJ 2014, 2, e601. [CrossRef]

60. Burnham, J.; Carlton, R.; Cherney, E.; Couret, G.; Eldridge, K.; Farzaneh, M.; Frazier, S.; Gorur, R.; Harness, R.; Shaffner, D.; et al. Preventive Measures to Reduce Bird-Related Power Outages-Part I: Electrocution and Collision. IEEE Trans. Power Deliv. 2004 19, 1843-1847. [CrossRef]

61. Google. Google Maps. 2020. Available online: https://www.google.com/maps/@42.4085098,-71.109201,15z (accessed on 4 May 2020).

62. Bergstra, J.; Yamins, D.; Cox, D.D. Making a Science of Model Search: Hyperparameter Optimization in Hundreds of Dimensions for Vision Architectures. PMLR 2013, 28, 115-123.

63. Turner, R.; Eriksson, D.; McCourt, M.; Kiili, J.; Laaksonen, E.; Xu, Z.; Guyon, I. Bayesian Optimization is Superior to Random Search for Machine Learning Hyperparameter Tuning: Analysis of the Black-Box Optimization Challenge 2020. arXiv 2021, arXiv:2104.10201. 